\title{
EXTENSIONS OF THE CAV(U) THEOREM FOR REPEATED GAMES WITH INCOMPLETE INFORMATION ON ONE SIDE.
}

\author{
FABIEN GENSBITTEL
}

\begin{abstract}
This work is devoted to extend several asymptotic results concerning repeated games with incomplete information on one side. The model we consider is a generalization of the classical model of Aumann and Maschler [4] to infinite action spaces and partial information. We prove an extension of the classical "Cav(u)" Theorem in this model for both the lower and upper value functions using two different methods: respectively a probabilistic method based on martingales and a functional one based on approximation schemes for viscosity solutions of Hamilton Jacobi equations similar to the dual differential approach of Laraki [25]. Moreover, we show that solutions of these two asymptotic problems provide $\mathcal{O}(1 / \sqrt{n})$-optimal strategies for both players in any game of length $n$. All these results are based on a compact approach, which consists in identifying a continuous-time problem defined on the time interval $[0,1]$ representing the "limit" of a sequence of finitely repeated games, as the number of repetitions is going to infinity. Finally, our results imply the existence of the uniform value of the infinitely repeated game whenever the value of the non-revealing game exists.
\end{abstract}

This work is devoted to generalize and extend several asymptotic results concerning zero-sum repeated games with incomplete information on one side. Our approach, known as compact approach, consists in identifying a continuous-time problem (in most known examples a differential game or a control problem) defined on the time interval $[0,1]$ which represents the "limit" of a sequence of finitely repeated games. These games are seen as discrete-time models defined on the time interval $[0,1]$, being piecewise constant on a partition whose mesh is going to zero as the number of repetitions is going to infinity.

The model we consider is an extension of the classical Aumann-Maschler model [4], where the informed player receives only a partial information about the state variable (i.e. a noisy signal) and where action spaces may be infinite. As a consequence, and since no regularity of the payoff functions is assumed, these games have in general no value. Our main result is the extension of the classical "Cav(u)" Theorem for both the lower and upper value functions in terms of the lower and upper value functions of the non-revealing game. Moreover, we identify two limit continuous-time problems, generalizing the compact problems mentioned in Sorin [29] (section 3.7.2 p.50). All the results are presented within the framework of finitely repeated games, but the extension to time-dependent payoff functions and to general evaluations (including discounted games) is possible (precise statements and details needed in order to adapt the proofs are given in section 5). These extensions are typical of the compact approach (see Cardaliaguet-Laraki-Sotin [14] for general results in this direction) and also relate our results to differential games with incomplete information (see Cardaliaguet-Rainer $[11,13]$ ).

The analysis of the asymptotic behavior of the lower value functions (or maxmin) of these games is based on probabilistic tools. The proofs rely on a representation formula for the lower value functions as the value functions of some discrete-time stochastic optimization problem over martingales that we call maximal variation problem. The maximizers of this problem represent the optimal processes of revelation for the informed player. This formula extends the one introduced in De Meyer [19] in a multidimensional setting and without assuming the existence of the value, since the class of financial exchange games considered in [19] is formally contained in the model described here. The concavification operator appearing in the limit is then expressed as the value function of a continuous-time stochastic control problem and we deduce from this representation that any limit point of a sequence of maximizers for the maximal variation problem is a maximizer of the control problem. Conversely, as for the finite case, any maximizer of the limit problem induces an $\mathcal{O}\left(\frac{1}{\sqrt{n}}\right)+\varepsilon$-optimal strategy for the informed player in the $n$-stages game for any $n$ (optimal strategies in this context being strategies for the players guaranteeing respectively the lower and upper value). Although some classical arguments are used, such as the splitting Lemma and the bound on the $L_{1}$-variation of a martingale, our proof also introduce new arguments in order to consider infinite action spaces. Moreover, we provide precise properties of the value functions and optimal strategies, some of them being new even for finite games, which may be interesting for their own sake.

The analysis of the upper value functions relies on the introduction of a dual game similar to the game introduced in De Meyer [18] for finite games and appearing more recently in De Meyer-Lehrer-Rosenberg [20] for a finite non-repeated game with partial information. We show that the idea of considering a PDE introduced in De Meyer [17] and already used in De Meyer-Rosenberg [21] and Laraki [25] to prove the classical "Cav(u)" Theorem for finite games can be adapted in our case. In particular, we extend the dual variational characterization in the viscosity sense of the concavification operator obtained in Laraki [25] on an infinitedimensional space of probabilities. This allows us to deduce from the asymptotic behavior of the upper value 
functions of the dual game the extension of the "Cav $(\mathrm{u})$ " Theorem for the upper value functions of the initial game. Moreover, these duality relationships allow us to construct, as for the lower values, $\mathcal{O}\left(\frac{1}{\sqrt{n}}\right)+\varepsilon-$ optimal strategies for the uninformed player in the $n$-stage game for any $n$.

These two asymptotic characterizations of the value functions, the first related to some continuous-time optimization problem over martingales and the second as a dual PDE problem, rely on very general arguments and provide tools that may be adapted in more general models of repeated game with incomplete information. Our choice to present these two methods together is based on the fact that they are both related to some continuous-time problem but also on the observation that the probabilistic method is not directly applicable for the asymptotic analysis of the upper value functions and reciprocally that the PDE method is not directly applicable for the asymptotic analysis of the lower value functions. Let us also mention that these two characterizations (the martingale optimization problem and the dual PDE formulation) also appear for second-order expansions in Gensbittel [23], where a different normalization is used for a particular subclass of games.

Finally, based on classical results on zero-sum repeated games, our results imply that both players have an $\varepsilon$-optimal strategy in the infinitely repeated game. We deduce from this observation the existence of the uniform value under the assumption, very similar to the Isaac's condition appearing in [11], that the non-revealing game has a value.

The paper is organized as follows. Section 1 describes the model and presents the main results. In section 2 , we study general properties of the value functions and strategies. In section 3, we prove the representation formula based on the problem of maximal variation. Then we prove the "Cav(u)" Theorem for the lower value functions and analyze the optimal strategies of the informed player. In section 4, we describe the model of the dual game, we prove the "Cav(u)" Theorem for the upper value functions, and we analyze the optimal strategies of the uninformed player. Section 5 discusses possible extensions and open questions. Section 6 is an appendix containing technical proofs and auxiliary results.

\section{Model And main Results.}

Our aim is to extend the classical model of Aumann-Maschler to infinite action spaces and to partial information by considering a zero-sum repeated game with incomplete information on one side in which the informed player does not observe the state variable directly but receives a stochastic signal whose distribution depends on the state variable. If a fixed finite set of signals and the distribution of signals given states were given, then this model could be reduced to the classical one (with full information on one side) by replacing states by signals and by modifying the payoff function accordingly. This would conduct to consider a different game for any finite set of signals and for any distribution. Moreover, this reduction is not helpful when considering infinite action spaces since to our knowledge previous proofs for the convergence of the upper value functions do not apply directly in this context even in the full information case (see Remark 2.1). Our approach is the following, we consider any measurable set of signals, allowing in particular for infinite sets, and we reduce the problem to the study of an auxiliary game where states are beliefs of the informed player. We study directly the value functions in this auxiliary model with compact state space and infinite action spaces which does not depend on the chosen set of signals. Let us also mention that the model with partial information, apart from being more general, can also be seen as a tool in order to study the lower value function for games with full information on one side and infinite action spaces through the representation formula given in Proposition 3.1.

In the following, for a finite set $K$, its cardinal is also denoted $K$. $|$.$| and \langle.,$.$\rangle denote respectively the usual$ Euclidean norm and scalar product on $\mathbb{R}^{K}$. For a measurable space $(E, \mathcal{E}), \Delta(E)$ denotes the set of probabilities over $E$. Topological sets are implicitly endowed with their Borel $\sigma$-fields, probabilities over a topological set with the weak*-topology, Cartesian products with the product topology and finite sets with the discrete topology.

The game with partial information. A zero-sum finitely repeated game with partial information on one side is represented by $(I, J, K, A,(S, \mathcal{S}))$. I, $J, K$ are respectively the action sets of the two players denoted $\mathrm{P} 1, \mathrm{P} 2$ and the state space. The set $K$ is assumed to be finite, but $I, J$ are only assumed to be Polish spaces (complete separable metric). A represents the payoff function and is a bounded measurable mapping

$$
A: I \times J \rightarrow \mathbb{R}^{K}
$$

where $A(i, j)^{k}$ is the payoff of $\mathrm{P} 1$ when actions $(i, j)$ are played and the state variable is $k$. $(S, \mathcal{S})$ is an auxiliary measurable space. At the beginning of the game, the pair $(k, s)$, where $k \in K$ is the state variable and $s \in S$ is a signal, is drawn by Nature using some probability $\pi \in \Delta(K \times S)$. P1 is informed privately of the realization of the random variable $s$ while P2 knows only the description of the game, including $\pi$. The only relevant information for $\mathrm{P} 1$ is the conditional law of $k$ given $s$ denoted $L(s) \in \Delta(K)$. Let $\mu$ denote the law of $L(s)$, and note that $\mu$ depends only on the joint law of $(k, s)$, which is known by $\mathrm{P} 2$. We can therefore reduce our analysis to a model with full information on one side where the state space $\Delta(K)$ is the set of beliefs of P1. This amounts to assume (see Lemma 2.1 below for a formal proof) that Nature selects at random $L \in \Delta(K)$ using the probability $\mu$ and informs $\mathrm{P} 1$ of his beliefs $L$. 
This observation leads us to consider the following reduced game, which is a classical game with incomplete information on one side, and whose definition is independent of the set of signals. The reduced game is defined by $\left(I, J, S_{K}, A\right)$. The state space is now $S_{K}:=\Delta(K)$ identified as the canonical simplex in $\mathbb{R}^{K}$. The state variable is a vector $L=\left(L^{1}, \ldots, L^{K}\right) \in S_{K}$ and is drawn by Nature at the beginning of the game using some probability $\mu \in \Delta\left(S_{K}\right)$. P1 is informed of the realization of the random variable $L$ while P2 knows only the description of the game. The $n$-times repeated game proceeds as follows. At round $q(q=1, . ., n), \mathrm{P} 1$ and P2 select simultaneously and independently an action $i_{q} \in I$ for $\mathrm{P} 1$ and $j_{q} \in J$ for P2 using some lotteries depending on their information and past observations. Actions are announced publicly after each round. Formally, a behavioral strategy $\sigma$ for $\mathrm{P} 1$ is a sequence $\left(\sigma_{1}, . ., \sigma_{n}\right)$ of transition probabilities depending on his information and past observations

$$
\sigma_{q}: S_{K} \times(I \times J)^{(q-1)} \rightarrow \Delta(I)
$$

where $\sigma_{q}\left(L, i_{1}, j_{1}, \ldots, i_{q-1}, j_{q-1}\right)$ denotes the lottery used to select the action $i_{q}$ played at round $q$ by P1 when the state is $L$ and the past history of the game is $\left(i_{1}, j_{1}, \ldots, i_{q-1}, j_{q-1}\right)$. Let $\Sigma_{n}$ be the set of behavioral strategies for P1. Similarly, a strategy $\tau$ for P2 is a sequence $\left(\tau_{1}, . ., \tau_{n}\right)$ of transition probabilities depending only on his past observations

$$
\tau_{q}:(I \times J)^{(q-1)} \rightarrow \Delta(J) .
$$

Let $\mathcal{T}_{n}$ denote the set of behavioral strategies for P2. Let us denote $\Gamma_{n}(\mu)$ the associated $n$-times repeated game. A triplet $(\mu, \sigma, \tau)$ induces by Tulcea's Theorem (see [28]) a unique probability $\Pi(\mu, \sigma, \tau) \in \Delta\left(S_{K} \times I^{n} \times J^{n}\right)$. Based on Lemma 2.1, the payoff function of $\mathrm{P} 1$ in $\Gamma_{n}(\mu)$ is given by

$$
g_{n}(\mu, \sigma, \tau):=\mathbb{E}_{\Pi(\mu, \sigma, \tau)}\left[\left\langle L, \frac{1}{n} \sum_{q=1}^{n} A\left(i_{q}, j_{q}\right)\right\rangle\right] .
$$

Let us define the lower and upper value function of the game $\Gamma_{n}(\mu)$, respectively the maximal and minimal payoffs $\mathrm{P} 1$ and $\mathrm{P} 2$ can guarantee:

$$
\begin{aligned}
& \underline{V}_{n}(\mu):=\sup _{\sigma \in \Sigma_{n}} \inf \mathbb{E}_{\tau \in \mathcal{T}_{n}} \mathbb{E}_{\Pi(\mu, \sigma, \tau)}\left[\left\langle L, \frac{1}{n} \sum_{q=1}^{n} A\left(i_{q}, j_{q}\right)\right\rangle\right], \\
& \bar{V}_{n}(\mu):=\inf _{\tau \in \mathcal{T}_{n}} \sup _{\sigma \in \Sigma_{n}} \mathbb{E}_{\Pi(\mu, \sigma, \tau)}\left[\left\langle L, \frac{1}{n} \sum_{q=1}^{n} A\left(i_{q}, j_{q}\right)\right\rangle\right] .
\end{aligned}
$$

The non-revealing game. In order to state the main convergence results, we need to introduce the value functions of the non-revealing game. The non-revealing game is a modification of $\Gamma_{1}(\mu)$ in which $\mathrm{P} 1$ is not informed of the realization $L$. The lower and upper value functions of this game are defined as follows:

$$
\begin{aligned}
& \underline{U(\mu)}:=\sup _{\sigma \in \Delta(I)} \inf _{\tau \in \Delta(J)} \mathbb{E}_{\Pi_{(\mu, \sigma, \tau)}}\left[\left\langle L, A\left(i_{1}, j_{1}\right)\right\rangle\right], \\
& \bar{U}(\mu):=\inf _{\tau \in \Delta(J)} \sup _{\sigma \in \Delta(I)} \mathbb{E}_{\Pi_{(\mu, \sigma, \tau)}}\left[\left\langle L, A\left(i_{1}, j_{1}\right)\right\rangle\right] .
\end{aligned}
$$

Using that $L$ and $\left(i_{1}, j_{1}\right)$ are independent in the above expectations, we can replace $L$ by its expectation, and it follows that

$$
\forall \mu \in \Delta\left(S_{K}\right), \underline{U}(\mu)=\underline{V}_{1}\left(\delta_{\mathbb{E}(\mu)}\right) \text { and } \bar{U}(\mu)=\bar{V}_{1}\left(\delta_{\mathbb{E}(\mu)}\right),
$$

where $\mathbb{E}(\mu):=\int_{S_{K}} x d \mu(x) \in S_{K}$ and $\delta_{x}$ denotes the Dirac mass at $x$.

The "Cav(u)" Theorems. We are now ready to state our main results. The first one concerns the lower value functions.

Theorem 1.1. For all $\mu \in \Delta\left(S_{K}\right)$,

$$
\operatorname{Cav}(\underline{U})(\mu) \leq \underline{V}_{n}(\mu) \leq \operatorname{Cav}(\underline{U})(\mu)+\frac{C_{A} \sqrt{K-1}}{\sqrt{n}},
$$

where $C_{A}:=\sup _{(i, j) \in I \times J}|A(i, j)|$.

If the proof of the first inequality is standard, the proof of the second one is based on the representation formula given in Proposition 3.1 and on the properties of the value function $\underline{V}_{1}$. Besides the above statement, this representation formula induces several results that may be interesting for their own sake: it allows to relate optimal strategies of P1 to optimal belief martingales (see Remark 3.1), provides a new expression for the recurrence formula (see Proposition 3.5), and is easily shown to converge to a continuous-time optimization problem whose value is $\operatorname{Cav}(\underline{U})$ (see Proposition 3.3 and 3.4 and section 5 for further extensions).

In a second part of the paper, based on the study of the dual game, we prove the following dual version of the "Cav(u)" Theorem for the upper value functions. 
Theorem 1.2. There exists a constant $C_{0}$, depending only on $K$ and $C_{A}$ such that for all $\mu \in \Delta\left(S_{K}\right)$

$$
\operatorname{Cav}(\bar{U})(\mu) \leq \bar{V}_{n}(\mu) \leq \operatorname{Cav}(\bar{U})(\mu)+C_{0} n^{-1 / 2} .
$$

The proof is based on a generalization of the dual differential approach of Laraki [25].

Let us finally mention that using classical results, the informed player can guarantee $\operatorname{Cav}(\underline{U})$ in the infinitely repeated game using the splitting Lemma (see Remark 3.2) and the uninformed player can guarantee $\lim \bar{V}_{n}=$ $\operatorname{Cav}(\bar{U})$ (see Remark 4.3). The next Corollary follows then directly from Theorems 1.1 and 1.2.

Corollary 1.1. If the non-revealing game has a value for all $x \in S_{K}$ (or equivalently for all $\mu \in \Delta\left(S_{K}\right)$ ), then

$$
\operatorname{Cav}(U)(\mu) \leq \underline{V}_{n}(\mu) \leq \bar{V}_{n}(\mu) \leq \operatorname{Cav}(U)(\mu)+C_{0} n^{-1 / 2}
$$

where $U=\underline{U}=\bar{U}$. Moreover, the uniform value of the infinitely repeated game exists.

\section{General properties.}

Identifications. We consider here the model with signals in the set $S$ and with initial probability $\pi \in \Delta(K \times S)$ described briefly at the beginning of the previous section. In this model, a behavioral strategy $\sigma$ for P1 is a sequence $\left(\sigma_{1}, . ., \sigma_{n}\right)$ such that

$$
\sigma_{q}: S \times(I \times J)^{(q-1)} \rightarrow \Delta(I),
$$

and the set of behavioral strategies for $\mathrm{P} 2$ is $\mathcal{T}_{n}$, as in the auxiliary game $\Gamma_{n}(\mu)$. A triplet $(\pi, \sigma, \tau)$ induces a unique probability $\Pi_{(\pi, \sigma, \tau)} \in \Delta\left(K \times S \times I^{n} \times J^{n}\right)$ and the payoff function of P1 is given by

$$
\mathbb{E}_{\Pi(\pi, \sigma, \tau)}\left[\frac{1}{n} \sum_{q=1}^{n} A\left(i_{q}, j_{q}\right)^{k}\right]
$$

The following result proves that there is no loss of generality in studying directly the auxiliary game $\Gamma_{n}(\mu)$.

Lemma 2.1. For all strategy $\sigma$ as defined above, there exists a strategy $\tilde{\sigma} \in \Sigma_{n}$ (i.e. which depends on s only through the conditional law of $k$ given $s$ denoted $L(s)$ ) giving the same payoff against any strategy $\tau$. Therefore the lower and upper value functions of the game depend only on $\pi$ through the distribution $\mu$ of $L(s)$ and are respectively equal to $\underline{V}_{n}(\mu)$ and $\bar{V}_{n}(\mu)$.

Proof. Note that by definition of $L(s)$, we have $L(s)=\mathbb{E}_{\pi}\left[e_{k} \mid s\right]$ where $\left(e_{1}, . ., e_{K}\right)$ denotes the canonical basis of $\mathbb{R}^{K}$. Moreover, given any pair of strategies $(\sigma, \tau)$, we have by construction that $L(s)=\mathbb{E}_{\Pi(\pi, \sigma, \tau)}\left[e_{k} \mid\right.$ $\left.s, i_{1}, j_{1}, . ., i_{q}, j_{q}\right]$ for all $q=1, . ., n$. Therefore, by taking the conditional expectation with respect to $\left.\left(s, i_{1}, j_{1}, . ., i_{q}, j_{q}\right)\right)$ in each term of the following sum, we obtain

$$
\mathbb{E}_{\Pi(\pi, \sigma, \tau)}\left[\frac{1}{n} \sum_{q=1}^{n} A\left(i_{q}, j_{q}\right)^{k}\right]=\mathbb{E}_{\Pi(\pi, \sigma, \tau)}\left[\frac{1}{n} \sum_{q=1}^{n}\left\langle e_{k}, A\left(i_{q}, j_{q}\right)\right\rangle\right]=\mathbb{E}_{\Pi(\pi, \sigma, \tau)}\left[\frac{1}{n} \sum_{q=1}^{n}\left\langle L(s), A\left(i_{q}, j_{q}\right)\right\rangle\right] .
$$

Notice then that P1 can generate a variable $\tilde{s}$ such that $(L(s), \tilde{s})$ has the same law as $(L(s), s)$ using an exogenous lottery. Define a strategy $\hat{\sigma}$ as $\hat{\sigma}_{q}\left(L(s), i_{1}, j_{1}, \ldots, i_{q-1}, j_{q-1}\right)=\sigma\left(\tilde{s}, i_{1}, j_{1}, \ldots, i_{q-1}, j_{q-1}\right)$. This does not define a behavioral strategy but a general strategy. Using Kuhn's Theorem (see [3]) in the auxiliary game $\Gamma_{n}(\mu)$ with state variable $L(s)$, there exists a strategy $\tilde{\sigma}$ equivalent to $\hat{\sigma}$ and which depend only on $L(s)$. Using equation (2.1), this strategy gives the same payoff as $\sigma$ against any $\tau$ since it induces the same law for $\left(L(s), i_{1}, j_{1}, . ., i_{n}, j_{n}\right)$. The second assertion is obvious.

The model with partial information clearly extends the classical model with full information on one side. More formally, note that for a fully informative signal $s$, the conditional law of $k$ given $s$ is the Dirac mass $\delta_{k}$. This leads naturally to introduce the following embedding function. At first, we can identify $K$ with the canonical basis $\left\{e_{k}, k=1, . ., K\right\}$ of $\mathbb{R}^{K}$. This induces naturally a linear isomorphism $h$ between $\Delta(K)$ and the compact face $\Delta\left(\left\{e_{k}, k=1, . ., K\right\}\right) \subset \Delta\left(S_{K}\right)$ of probabilities over $\mathbb{R}^{K}$ supported by $\left\{e_{k}, k=1, . ., K\right\}$. Precisely,

$$
h: p=\left(p^{k}\right)_{k=1, \ldots, K} \in \Delta(K) \longrightarrow \sum_{k=1}^{K} p^{k} \delta_{e_{k}},
$$

where $\delta_{e_{k}}$ is the Dirac mass in $e_{k}$. Using this identification, it is easily seen that the function $p \rightarrow \underline{V}_{n}(h(p))$ defined on $\Delta(K)$ is the lower value function of the $n$-stages game in which P1 is informed of the state variable $k$ of law $p$. More can be said about the value of the non-revealing game, since it depends only on the law of the state variable $k$ and not on the law of signals. Precisely, define

$$
\begin{gathered}
\forall p \in \Delta(K), \underline{u}(p):=\underline{U}(h(p))=\underline{V}_{1}\left(\delta_{p}\right), \\
\bar{u}(p):=\bar{U}(h(p))=\bar{V}_{1}\left(\delta_{p}\right) .
\end{gathered}
$$

$\underline{u}(p)$ is the lower value function of the non-revealing game associated to a state variable $k$ of law $p$. 
Remark 2.1. The lower value function $\underline{V}_{1}$ associated with the model with partial information has the following important property that will be used in the next section. This concave and Lipschitz function extends the two usual value functions used in the classical Aumann-Maschler model. On one hand, looking at $\underline{V}_{1}$ on the set of extreme points of $\Delta\left(S_{K}\right)$, i.e. Dirac masses, one recovers the function of the classical non-revealing game $\underline{u}$. On the other hand, looking at $\underline{V}_{1}$ on the compact face of distributions supported by the elements of the canonical basis of $\mathbb{R}^{K}$, one recovers the value of the one-shot game with full information on one side. This property, together with the fact that $\underline{V}_{1}$ is Lipschitz (see the next Proposition), is a key argument in order to prove the "Cav(u)" Theorem without using a finiteness assumption on I (as for example in [26] Lemma 2.5 p.187).

Properties of the value functions. The following results are classical in zero-sum games. Notice however that the proof of the Lipschitz property cannot be derived from the usual argument used in the model with full information and relies on a probabilistic representation for the Wasserstein distance.

Proposition 2.1. The functions $\underline{V}_{n}$ and $\bar{V}_{n}$ are concave on $\Delta\left(S_{K}\right)$, nondecreasing for the convex order ${ }^{1}$ (denoted $\preceq$ ), and Lipschitz-continuous of constant $C_{A}$ with respect to the Wasserstein distance of order 1 (denoted $\bar{d}_{W}$, see definition 6.1).

Proof. Let us prove the nondecreasing property. Suppose that $\mu_{1} \preceq \mu_{2}$, and let $\left(Y_{1}, Y_{2}\right)$ be a martingale such that $Y_{i} \sim \mu_{i}$ for $i=1,2$. Let $\sigma$ be a strategy in $\Gamma_{n}\left(\mu_{1}\right)$. Assuming that the state variable is $Y_{2}, \mathrm{P} 1$ can generate $Y_{1}$ conditionally on $Y_{2}$ and then play the strategy $\sigma\left(Y_{1},.\right)$ in the game $\Gamma_{n}\left(\mu_{2}\right)$. This defines a generalized strategy which is equivalent to a behavioral strategy (see Aumann [3]) in $\Sigma_{n}$ and using the martingale property, the induced payoff in $\Gamma_{n}\left(\mu_{2}\right)$ against any strategy $\tau$ is

$$
\mathbb{E}\left[\left\langle Y_{2}, \frac{1}{n} \sum_{q=1}^{n} A\left(i_{q}, j_{q}\right)\right\rangle\right]=\mathbb{E}\left[\left\langle Y_{1}, \frac{1}{n} \sum_{q=1}^{n} A\left(i_{q}, j_{q}\right)\right\rangle\right]=g_{n}\left(\mu_{1}, \sigma, \tau\right),
$$

implying that P1 can guarantee at least the same quantity in $\Gamma_{n}\left(\mu_{2}\right)$ than in $\Gamma_{n}\left(\mu_{1}\right)$. For the Lipschitz property, let $\mu_{1}, \mu_{2} \in \Delta\left(S_{K}\right)$ and $\left(Y_{1}, Y_{2}\right)$ be random variables such that $Y_{i} \sim \mu_{i}$ for $i=1,2$ and $\mathbb{E}\left[\left|Y_{1}-Y_{2}\right|\right]=d_{W}\left(\mu_{1}, \mu_{2}\right)$. Given a strategy $\sigma$ in $\Gamma_{n}\left(\mu_{1}\right)$, playing $\sigma\left(Y_{1},.\right)$ defines also a strategy $\tilde{\sigma}$ in $\Gamma_{n}\left(\mu_{2}\right)$ using the same argument as above. Given any strategy $\tau \in \mathcal{T}_{n}$, we can assume that the random variables $\left(Y_{1}, Y_{2}\right)$ and $\left(i_{q}, j_{q}\right) \in I \times J$ are defined on the same probability space, and using Cauchy-Schwarz inequality

$$
g_{n}\left(\mu_{1}, \sigma, \tau\right)-g_{n}\left(\mu_{2}, \tilde{\sigma}, \tau\right)=\mathbb{E}\left[\left\langle Y_{1}-Y_{2}, \frac{1}{n} \sum_{q=1}^{n} A\left(i_{q}, j_{q}\right)\right\rangle\right] \leq C_{A} d_{W}\left(\mu_{1}, \mu_{2}\right),
$$

and the result follows. For the concavity, using Kuhn's Theorem, the game in mixed strategies has the same value functions, and can be seen as a one-shot game with incomplete information on one side. The result follows therefore from Theorem 1.1 p.183 in [26].

Reduced strategies. We introduce the notion of reduced strategies, which are simply strategies that do not depend on P2's past actions. These results will only be used in the beginning of section 4 and can be omitted at a first reading.

A reduced strategy $\tau$ for $\mathrm{P} 2$ is a sequence $\left(\tau_{1}, \ldots, \tau_{n}\right)$ of transition probabilities $\tau_{q}: I^{q-1} \rightarrow \Delta(J)$. A reduced strategy for $\mathrm{P} 1$ is a sequence $\sigma=\left(\sigma_{1}, \ldots, \sigma_{n}\right)$ of transition probabilities $\sigma_{q}: S_{K} \times I^{q-1} \rightarrow \Delta(I)$. Let $\Sigma_{n}^{R}$ (resp. $\mathcal{T}_{n}^{R}$ ) the set of reduced behavioral strategies of P1 (resp. P2). Given a reduced strategy $\sigma$ of $\mathrm{P} 1$, the pair $(\mu, \sigma)$ induces a probability on $S_{K} \times I^{n}$. The next Proposition implies that an optimal strategy in the game restricted to reduced strategies is still optimal in the initial game.

Proposition 2.2. If a strategy guarantees the quantity $C$ in the game $\Gamma_{n}(\mu)$ where the strategy sets of the players are restricted to reduced strategies, then it guarantees $C$ in the initial game. Moreover, for any strategy of P2, there exists a reduced strategy giving the same payoff against all reduced strategies of P1.

Proof. This proof is directly adapted from [18], and is reproduced here for the sake of completeness since it is very short. For any $\tau \in \mathcal{T}_{n}$, there exists a reduced strategy $\widehat{\tau}$ giving the same payoff as $\tau$ against any reduced strategy of P1. The strategy $\widehat{\tau}$ proceeds as follows : at step $q$, P2 does not remind his past actions $\left(j_{1}, . ., j_{q-1}\right)$, but using past actions of $\mathrm{P} 1$, he generates a virtual history $\left(i_{r}, \widehat{j_{r}}\right)_{r=1, \ldots, q-1}$ by choosing $\widehat{j_{r}}$ with the probability $\tau\left(i_{1}, \widehat{j_{1}}, . ., i_{r-1}, \widehat{j_{r-1}}\right)$. He selects then at stage $q$ an action $j_{q}$ with the probability $\tau\left(\left(i_{r}, \widehat{j_{r}}\right)_{r=1, . ., q-1}\right)$. Since the action of $\mathrm{P} 1$ does not depend on past actions of $\mathrm{P} 2$, the conditional distribution of $\left(i_{q}, j_{q}\right)$ given $\left(L, i_{1}, . ., i_{q-1}\right)$ is the same as if $\mathrm{P} 2$ was using $\tau$, and so is the conditional expected payoff at stage $q$. The situation is not symmetric for P1, because to generate a virtual history of the past actions of P2, he has to know which strategy P2 is using. However, given $\tau^{*} \in \mathcal{T}_{n}^{R}$, the same argument shows that for all $\sigma \in \Sigma_{n}$, there exists a reduced strategy $\widehat{\sigma}$ giving the same payoff as $\sigma$ against the fixed strategy $\tau^{*}$, which allows to conclude.

\footnotetext{
${ }^{1}$ Recall that $\mu_{1} \preceq \mu_{2}$ if there exists a martingale $X_{1}, X_{2}$ such that $X_{i}$ is $\mu_{i}$ distributed for $i=1,2$ (see Blackwell [7]).
} 


\section{Problem of maximal VARiation AND LOWER VAlue FUnCtions.}

We introduce now a general optimization problem called maximal variation problem that will lead to a continuous-time stochastic control problem describing the limits of optimal strategies of the informed player. The first main result is that the lower value function (or maxmin) of the game $\Gamma_{n}(\mu)$ is equal to the value function of the maximal variation problem (Proposition 3.1). This generalizes therefore the former result of De Meyer [19] in a multi-dimensional context and without the assumption of existence of the value. Using this representation, we extend the classical "Cav(u)" Theorem of Aumann-Maschler for the lower value functions. The limiting function given by this Theorem is then shown to be the value function of an optimization problem over continuous-time martingale distributions and we prove that the limit of any convergent sequence of maximizers for the maximal variation problem is a maximizer of the limit or "compact" problem (Proposition 3.3). On the other hand, maximizers of this compact problem provide $\mathcal{O}(\sqrt{n})$-optimal strategies in the games $\Gamma_{n}$ (Proposition 3.4).

Proposition 3.1 allows to prove easily and rigorously convergence to the continuous-time limit problem. As mentioned in the introduction, this result relates our asymptotic results to differential games with asymmetric information (see also section 5). Note also that the same kind of asymptotic analysis have been derived from this representation formula in order to obtain second-order asymptotic expansions in De Meyer [19] and Gensbittel [23]. Moreover, besides the present applications, we believe that this method is of interest for its own sake, and can be adapted in much more general contexts in which one player is always more informed (see the last point in section 5).

The martingale of beliefs. As usual when computing a best reply to some strategy of the informed player P1, we "assume" that P2 knows which strategy P1 is using and updates his beliefs on the state variable $L$ given his observations using Bayes' rules. This leads to introduce a sequence of virtual beliefs of P2 about the state variable which depends on the strategy used by both players and is called the a posteriori martingale. In a more general context, beliefs of P2 should be represented by the conditional distribution of the state variable over $\mathbb{R}^{K}$ given his information. In our case, since we already work with an auxiliary game in which payoffs are linear, we shall only consider the process of expected beliefs ${ }^{2}$ of P2. Precisely, let us define the expected belief of $\mathrm{P} 2$ as the expected value of $L$ given his information. Available information after round $q$ is represented by the $\sigma$-field $\mathcal{F}_{q}=\sigma\left(i_{1}, j_{1}, \ldots, i_{q}, j_{q}\right)$ of past observations. Formally, the process of expected beliefs is the martingale

$$
L_{q}:=\mathbb{E}_{\Pi(\mu, \sigma, \tau)}\left[L \mid \mathcal{F}_{q}\right],
$$

and the law of this martingale depends on the pair of strategies $(\sigma, \tau)$. This martingale has length $n$ and its final value $L_{n}$ is by construction dominated (in the sense of convex ordering) by $\mu$. The initial value is by convention $L_{0}:=\mathbb{E}_{\mu}[L]$ and we will consider the variable $L_{n+1}:=L$ as the terminal value of the martingale ${ }^{3}$, which follows the law $\mu$.

The maximal variation problem. Let us introduce some notations and definitions.

Notation 3.1. Let $E$ be a Borel space and $X$ be an E-valued random variable defined on some probability space $(\Omega, \mathcal{F}, \mathbb{P})$. Then $\llbracket X \rrbracket$ denotes the law of $X$ and given a $\sigma$-field $\mathcal{G} \subset \mathcal{F}, \llbracket X \mid \mathcal{G} \rrbracket$ denotes a version of the conditional law of $X$ given $\mathcal{G}$, hence a $\mathcal{G}$ measurable random variable with values in $\Delta(E)$ (see Proposition 7.26 in [6]).

Definition 3.1. For $\mu \in \Delta\left(S_{K}\right), \mathcal{M}_{n}(\mu)$ denotes the subset of $\Delta\left(S_{K}^{n}\right)$ formed by the laws of $S_{K}$-valued martingales $\left(L_{1}, . ., L_{n}\right)$ such that the final distribution $\llbracket L_{n} \rrbracket$ is dominated by $\mu$. By convention, for any such martingale $L_{0}:=\mathbb{E}\left[L_{1}\right]$.

Definition 3.2. We define the $\underline{V}_{1}$-variation on the set $\mathcal{M}_{n}(\mu)$ by

$$
\Psi_{n}\left(\left(L_{q}\right)_{q=1, . ., n}\right):=\frac{1}{n} \mathbb{E}\left[\sum_{q=1}^{n} \underline{V}_{1}\left(\llbracket L_{q} \mid L_{m}, 0 \leq m \leq q-1 \rrbracket\right)\right] .
$$

The problem of maximal $\underline{V}_{1}$-variation is then

$$
\bar{\Psi}_{n}(\mu):=\sup _{\mathcal{M}_{n}(\mu)} \Psi_{n}
$$

The following Proposition is the keystone of our proof of the "Cav $(\mathrm{u})$ " Theorem for the lower value functions.

\section{Proposition 3.1.}

$$
\forall \mu \in \Delta\left(S_{K}\right), \quad \underline{V}_{n}(\mu)=\bar{\Psi}_{n}(\mu) .
$$

As it will be convenient to work with martingales with respect to arbitrary filtrations, let us introduce the following definition.

\footnotetext{
${ }^{2} \mathrm{~A}$ direct computation shows that in a game with signals, the expected belief we define here is exactly the belief of the uninformed player about the "true" state variable $k$ which is not appearing anymore in our description.

${ }^{3}$ Note that it is always possible to add an additional variable having law $\mu$ to a martingale whose final distribution is dominated by $\mu$.
} 
Definition 3.3. For $\mu \in \Delta\left(S_{K}\right), \mathfrak{M}_{n}(\mu)$ is the collection of martingales $\left(L_{q}, \mathcal{H}_{q}\right)_{q=1, . ., n}$ of length $n$, defined on some filtered probability space $\left(\Omega, \mathcal{A},\left(\mathcal{H}_{q}\right)_{q=0, . ., n}, \mathbb{P}\right)$, and whose final distribution $\llbracket L_{n} \rrbracket$ is dominated by $\mu$. By convention, we always set $\mathcal{H}_{0}:=\{\Omega, \emptyset\}$.

Note that the set $\mathcal{M}_{n}(\mu)$ introduced in Definition 3.1 is the set of laws of martingales in $\mathfrak{M}_{n}(\mu)$. The following property results directly from the concavity of $\underline{V}_{1}$ and shows that the maximal variation problem can be extended to the set $\mathfrak{M}_{n}(\mu)$ without modifying its value.

Lemma 3.1. For all $\mu \in \Delta\left(S_{K}\right)$, we have

$$
\bar{\Psi}_{n}(\mu)=\underset{\left(L_{q}, \mathcal{H}_{q}\right)_{q=1, \ldots, n} \in \mathfrak{M}_{n}(\mu)}{\sup } \frac{1}{n} \mathbb{E}\left[\sum_{q=1}^{n} \underline{V}_{1}\left(\llbracket L_{q} \mid \mathcal{H}_{q-1} \rrbracket\right)\right] .
$$

Proof. It is clearly sufficient to prove that $\bar{\Psi}_{n}(\mu)$ is not lower than the right-hand side of (3.3). Let $\left(L_{q}, \mathcal{H}_{q}\right)_{q=1, . ., n} \in \mathfrak{M}_{n}(\mu)$. Since $\underline{V}_{1}$ is concave and $d_{W}$-Lipschitz, it follows from Jensen's inequality (see Lemma 6.1) that for all $q=1, . ., n$

$$
\underline{V}_{1}\left(\llbracket L_{q} \mid \mathcal{H}_{q-1} \rrbracket\right) \leq \underline{V}_{1}\left(\llbracket L_{q} \mid L_{0}, . ., L_{q-1} \rrbracket\right) .
$$

The proof follows then by summation over $q$.

In order to prove Proposition 3.1, we will also need the following two Lemmas that are classical measurable selection results.

Lemma 3.2. For all $\varepsilon>0$, there exists a measurable function

$$
\varphi_{\varepsilon}: S_{K} \times \Delta\left(S_{K}\right) \times[0,1] \rightarrow I,
$$

such that the strategy induced by $i_{1}=\varphi_{\varepsilon}(L, \mu, U)$, where $U$ is some uniform random variable on $[0,1]$ independent from $L$, is $\varepsilon$-optimal in the game $\Gamma_{1}(\mu)$ for all $\mu \in \Delta\left(S_{K}\right)$.

The proof of this first Lemma is standard and therefore postponed to section 6 . Let us denote $\Delta\left(I \times S_{K}\right)$ the set of joint probability distributions on $I \times S_{K}$. Note that a pair $\left(\sigma_{1}, \mu\right)$ where $\sigma_{1}$ is a strategy of P1 in the game $\Gamma_{1}(\mu)$ defines naturally a probability in $\Delta\left(I \times S_{K}\right)$ that will be denoted $\pi\left(\sigma_{1}, \mu\right)$.

Lemma 3.3. For all $\varepsilon>0$, there exists a universally measurable function

$$
\tau_{\varepsilon}: \Delta\left(I \times S_{K}\right) \rightarrow \Delta(J)
$$

such that for all $\left(\sigma_{1}, \mu\right)$

$$
\mathbb{E}_{\Pi\left(\mu, \sigma_{1}, \tau_{\varepsilon}\left(\pi\left(\sigma_{1}, \mu\right)\right)\right)}\left[\left\langle L, A\left(i_{1}, j_{1}\right)\right\rangle\right] \leq \underline{V}_{1}(\mu)+\varepsilon .
$$

Proof. Endow the sets $\Delta\left(I \times S_{K}\right)$ and $\Delta(J)$ with the usual weak* topology. The application

$$
(\pi, \tau) \rightarrow \int\langle x, T(i, j)\rangle d \pi(i, x) \otimes \tau(j)
$$

is jointly measurable. If $\mu$ denotes the marginal law on $S_{K}$ induced by $\pi$, the set

$$
\left\{(\pi, \tau): \int\langle x, T(i, j)\rangle d \pi(i, x) \otimes \tau(j) \leq \underline{V}_{1}(\mu)+\varepsilon\right\}
$$

is therefore a Borel subset of $\Delta\left(I \times S_{K}\right) \times \Delta(J)$. The existence of an $\varepsilon$-optimal universally measurable selection $\tau_{\varepsilon}$ follows therefore from Von Neumann's selection Theorem (see e.g. Proposition 7.49 in [6]).

of Proposition 3.1. The first part of this proof is an extension of the one appearing in [19]. However, the second part is different since we consider the lower value of the game and not the value, and requires the assumption that the actions sets are Polish. Let us start with an $\varepsilon$-maximizer $\left(L_{q}\right)_{q=1, \ldots, n}$ for the problem $\bar{\Psi}_{n}(\mu)$. Formally, since only the law of the chosen martingale is relevant, we can assume (see Theorem 6.1) that there exists a sequence $U_{1}, . ., U_{n}$ of independent random variables uniformly distributed on $[0,1]$ and independent from $L$, and a sequence of measurable functions $\left(f_{1}, . ., f_{n}\right)$ such that $\left(L_{1}, . ., L_{n}, L\right)$ is a martingale and

$$
L_{q}=f_{q}\left(L, U_{1}, . ., U_{q}\right) \text { for } q=1, . . n .
$$

From the previous Lemma, we can define P1's strategy $\sigma$ as follows: given $\eta>0$, and a sequence $\left(Y_{1}, . ., Y_{n}\right)$ of independent random variables uniformly distributed on $[0,1]$ and independent from $\left(L, U_{1}, . ., U_{n}\right)$, the action $i_{q}$ of $\mathrm{P} 1$ at stage $q$ is

$$
i_{q}\left(L_{1}, . ., L_{q}, Y_{q}\right):=\varphi_{\eta}\left(L_{q}, \llbracket L_{q} \mid L_{1}, . ., L_{q-1} \rrbracket, Y_{q}\right) .
$$

This does not define directly a behavioral strategy, since P1 has to remember at each stage the value of the auxiliary variables $L_{q}$ (or $\left.U_{q}\right)$. But this mixed strategy defines a joint law on $\left(L, i_{1}, . ., i_{n}\right)$ which can always be disintegrated in a behavioral strategy that does not depend on P2's actions. We can clearly keep the above representation of P1's strategy to compute his payoff against some strategy $\tau$, even if it is not expressed as a behavioral strategy, since these computations depend only on the induced law on $\left(L, i_{1}, . . i_{n}\right)$. Without loss 
of generality, given a sequence $\left(W_{1}, . ., W_{n}\right)$ of independent random variables uniformly distributed on $[0,1]$ and independent of $\left(L, U_{1}, Y_{1}, . ., U_{n}, Y_{n}\right)$, we can assume that the strategy $\tau$ is given by a sequence $g_{1}, . ., g_{n}$ of measurable functions and that the action of P2 at stage $q$ is (see Theorem 6.1)

$$
j_{q}=g_{q}\left(i_{1}, j_{1}, . ., i_{q-1}, j_{q-1}, W_{q}\right) \text {. }
$$

Let us define the filtration $\left(\mathcal{H}_{q}\right)_{q=1, . ., n}$ by $\mathcal{H}_{q}=\sigma\left(L_{1}, Y_{1}, W_{1}, . ., L_{q}, Y_{q}, W_{q}\right)$. With this notation, variables $i_{q}, j_{q}$ are $\mathcal{H}_{q}$-measurable and we have by construction the following equalities between the conditional laws for all $q=1, . ., n$

$$
\llbracket L\left|\mathcal{H}_{q} \rrbracket=\llbracket L\right| L_{1}, . ., L_{q} \rrbracket, \quad \llbracket L_{q}\left|\mathcal{H}_{q-1} \rrbracket=\llbracket L_{q}\right| L_{1}, . ., L_{q-1} \rrbracket .
$$

The conditional payoff at round $q$ given the past actions is

$$
\begin{aligned}
& \mathbb{E}\left[\left\langle L, A\left(i_{q}, j_{q}\right)\right\rangle \mid i_{1}, j_{1}, . ., i_{q-1}, j_{q-1}\right]=\mathbb{E}\left[\mathbb{E}\left[\left\langle L, A\left(i_{q}, j_{q}\right)\right\rangle \mid \mathcal{H}_{q}\right] \mid i_{1}, j_{1}, . ., i_{q-1}, j_{q-1}\right] \\
& \quad=\mathbb{E}\left[\left\langle L_{q}, A\left(i_{q}, j_{q}\right)\right\rangle \mid i_{1}, j_{1}, . ., i_{q-1}, j_{q-1}\right]=\mathbb{E}\left[\mathbb{E}\left[\left\langle L_{q}, A\left(i_{q}, j_{q}\right)\right\rangle \mid \mathcal{H}_{q-1}\right] \mid i_{1}, j_{1}, . ., i_{q-1}, j_{q-1}\right],
\end{aligned}
$$

where the second equality follows from the linearity of the payoff. Finally the conditional expectation given $\mathcal{H}_{q-1}$ above is exactly the payoff in a one-shot game where the law of the state variable is $\llbracket L_{q} \mid L_{1}, . ., L_{q-1} \rrbracket$ and the joint conditional law of $\llbracket L_{q}, i_{q} \mid L_{1}, . ., L_{q-1} \rrbracket$ has been constructed so that

$$
\mathbb{E}\left[\left\langle L_{q}, A\left(i_{q}, j_{q}\right)\right\rangle \mid \mathcal{H}_{q-1}\right] \geq \underline{V}_{1}\left(\llbracket L_{q} \mid L_{1}, . ., L_{q-1} \rrbracket\right)-\eta .
$$

Summing up these inequalities proves that $\underline{V}_{n}(\mu) \geq \bar{\Psi}_{n}(\mu)-(\varepsilon+\eta)$ and we obtain a first inequality by sending $\varepsilon$ and $\eta$ to zero.

It remains to prove the reverse inequality. Let us fix a pair $(\mu, \sigma)$ where $\sigma$ is a behavioral strategy for P1 in $\Gamma_{n}(\mu)$ and some $\varepsilon>0$. We will construct a strategy $\tau$ for P2 by induction such that for all $q=1, . ., n$ the expected payoff at round $q$ is not greater than

$$
\mathbb{E}_{\Pi(\mu, \sigma, \tau)}\left[\underline{V}_{1}\left(\llbracket L_{q} \mid i_{1}, j_{1}, . ., i_{q-1}, j_{q-1} \rrbracket\right)\right]+\varepsilon,
$$

where $L_{q}$ is defined by $\mathbb{E}_{\Pi(\mu, \sigma, \tau)}\left[L \mid i_{1}, j_{1}, . ., i_{q-1}, j_{q-1}, i_{q}\right]$. Note that adding $j_{q}$ in the conditional expectation defining $L_{q}$ does not change its value, which does not depend on $\tau_{q}$. Suppose that $\left(\tau_{1}, . ., \tau_{q-1}\right)$ is already constructed. Since $\left(\mu, \sigma, \tau_{1}, . ., \tau_{q-1}\right)$ defines a joint law on $\left(L, i_{1}, . ., i_{q}, j_{1}, . ., j_{q-1}\right)$ that will be denoted $\mathbb{P}$, the conditional expectation $L_{q}$ is well-defined and $\mathbb{P}$-almost surely equal to a Borel mapping $h_{q}\left(i_{q}, i_{1}, j_{1}, \ldots, i_{q-1}, j_{q-1}\right)$. The disintegration Theorem implies the existence of a $\Delta\left(S_{K} \times I\right)$-valued Borel mapping $M_{q}\left(i_{1}, j_{1}, \ldots, i_{q-1}, j_{q-1}\right)$ which is a version of the conditional law $\llbracket L_{q}, i_{q} \mid i_{1}, j_{1}, \ldots, i_{q-1}, j_{q-1} \rrbracket$. Using Lemma 3.3, the mapping $\tau_{\varepsilon}$ being universally measurable, there exists a Borel mapping $\tilde{\tau}_{\varepsilon}$ which is almost surely equal to $\tau_{\varepsilon}$ with respect to the law of the random variable $\llbracket L_{q}, i_{q} \mid i_{1}, j_{1}, \ldots, i_{q-1}, j_{q-1} \rrbracket$ (or the image probability of $\mathbb{P}$ induced by the mapping $M_{q}$ which depends only on variables known by P2). The strategy $\tau_{q}=\tilde{\tau}_{\varepsilon}\left(M_{q}\left(i_{1}, j_{1}, \ldots, i_{q-1}, j_{q-1}\right)\right)$ has then the required properties. The overall payoff of $\mathrm{P} 2$ is therefore not greater than

$$
\mathbb{E}_{\Pi(\mu, \sigma, \tau)}\left[\frac{1}{n} \sum_{q=1}^{n} \underline{V}_{1}\left(\llbracket L_{q} \mid i_{1}, j_{1}, . ., i_{q-1}, j_{q-1} \rrbracket\right)\right]+\varepsilon \leq \bar{\Psi}_{n}(\mu)+\varepsilon,
$$

which concludes the proof since $\varepsilon$ was arbitrary.

Remark 3.1. The above proof implies that any $\varepsilon$-maximizer of $\bar{\Psi}_{n}(\mu)$ induces an $\varepsilon+\eta$-optimal reduced strategy for P1 in $\Gamma_{n}(\mu)$ through the formula (3.4). On the other hand, it also implies that if a strategy of P1 is $\varepsilon$-optimal in $\Gamma_{n}(\mu)$ and does not depend on the actions of PQ, then the induced martingale of expected beliefs is $\varepsilon$-optimal for $\bar{\Psi}(\mu)$ independently of the strategy of P2.

The "Cav(u)" Theorem. We are now able to prove the classical "Cav(u)" Theorem of Aumann-Maschler in this context. Let us recall, the classical bound on the $L_{1}$-variation of $S_{K}$-valued martingales.

Lemma 3.4. For all martingale $\left(L_{1}, \ldots, L_{n}\right)$ with values in $S_{K}$,

$$
\mathbb{E}\left[\sum_{q=1}^{n}\left|L_{q}-L_{q-1}\right|\right] \leq \sqrt{K-1} \sqrt{n} .
$$

Proof. See Lemma 2.1 p. 186 in [26].

of Theorem 1.1. The proof of the first inequality is well-known, see for example Proposition 2.8 p.188 in [26]. The second inequality follows from Proposition 2.1. Using the Lipschitz property of $\underline{V}_{1}$ we have

$$
\forall \mu \in \Delta\left(S_{K}\right), \quad \underline{V}_{1}(\mu) \leq \underline{U}(\mu)+C_{A} d_{W}\left(\mu, \delta_{\mathbb{E}(\mu)}\right) .
$$


Note that for any variable $X$ of law $\mu, d_{W}\left(\mu, \delta_{\mathbb{E}(\mu)}\right)=\mathbb{E}[|X-\mathbb{E}[X]|]$. Applying the preceding inequality in the expression of $\Psi_{n}\left(\left(L_{q}\right)_{q=1, . ., n}\right)$ implies, with $L_{n+1}$ a terminal variable of law $\mu$ added to the martingale:

$$
\begin{aligned}
n \Psi_{n}\left(\left(L_{q}\right)_{q=1, . ., n}\right) & \leq \mathbb{E}\left[\sum_{q=1}^{n} \underline{U}\left(\llbracket L_{q} \mid L_{1}, . ., L_{q-1} \rrbracket\right)\right]+C_{A} \mathbb{E}\left[\sum_{q=1}^{n}\left|L_{q}-L_{q-1}\right|\right] \\
& \leq \mathbb{E}\left[\sum_{q=1}^{n} \underline{U}\left(\llbracket L_{n+1} \mid L_{1}, . ., L_{q-1} \rrbracket\right)\right]+C_{A} \sqrt{n} \sqrt{K-1} \\
& \leq \mathbb{E}\left[\sum_{q=1}^{n} \operatorname{Cav}(\underline{U})\left(\llbracket L_{n+1} \mid L_{1}, . ., L_{q-1} \rrbracket\right)\right]+C_{A} \sqrt{n} \sqrt{K-1} \\
& \leq \sum_{q=1}^{n} \operatorname{Cav}(\underline{U})\left(\llbracket L_{n+1} \rrbracket\right)+C_{A} \sqrt{n} \sqrt{K-1} \\
& \leq n \operatorname{Cav}(\underline{U})(\mu)+C_{A} \sqrt{n} \sqrt{K-1} .
\end{aligned}
$$

The second line follows from the fact that $\underline{U}(\nu)$ depends only the barycenter of $\nu$ (cf. (1.1)) and from the martingale property. The third line follows from the definition of the concavification operator. The fourth one follows from Jensen's inequality (Lemma 6.1), which applies here since $\underline{U}$ is weakly continuous on the weakly compact set $\Delta\left(S_{K}\right)$ and bounded, implying that $\operatorname{Cav}(\underline{U})$ is weakly upper semi-continuous and bounded (see e.g. Lemma 26.13 in $[15])$.

Remark 3.2. Recall that Proposition 2.8 p.188 in [26] provides a strategy which guarantees Cav $(\underline{U})(\mu)$ in $\Gamma_{n}(\mu)$ for all $n$ and also in the infinitely repeated game. From the preceding Theorem, this strategy is asymptotically optimal for P1 in $\Gamma_{n}(\mu)$.

The limit continuous-time optimization problem. The following continuous-time probabilistic formulation is obtained directly from the definition of the concavification operator and already appears in [29] (section 3.7.2.a. p.50) as the heuristic compact limit for finite games. It is also reminiscent from the result given in Theorem 3.1 of [11] for differential games with incomplete information with time dependent payoffs (see section 5 for possible extensions in this direction).

Proposition 3.2. For all $\mu \in \Delta\left(S_{K}\right)$,

$$
\operatorname{Cav}(\underline{U})(\mu)=\sup _{\llbracket\left(X_{t}\right)_{t \in[0,1]} \rrbracket \mathcal{M}(\preceq \mu)} \mathbb{E}\left[\int_{0}^{1} \underline{u}\left(X_{t}\right) d t\right],
$$

where $\mathcal{M}(\preceq \mu)$ is the set of laws of càdlàg martingales $\left(X_{t}\right)_{t \in[0,1]}$ whose final distribution $\llbracket X_{1} \rrbracket$ is dominated by $\mu$. Moreover, $X$ is a maximizer if and only if for all $s$ in $[0,1)$

$$
\mathbb{E}\left[\underline{u}\left(X_{s}\right)\right]=\operatorname{Cav}(\underline{U})(\mu) .
$$

Proof. Note at first that any martingale in $\mathcal{M}(\preceq \mu)$ has trajectories in the set $S_{K}$ with probability 1 . Moreover, $X$ being a martingale, we have for all $s \leq t, \llbracket X_{s} \rrbracket \preceq \llbracket X_{t} \rrbracket$ using the definition of convex order. From the definition of $\underline{u}$, for all $s \in[0,1]$, we have $\underline{u}\left(X_{s}\right)=\underline{U}\left(\llbracket X_{1} \mid \mathcal{F}_{s}^{X} \rrbracket\right)$ almost surely, where $\mathcal{F}^{X}$ denotes the filtration generated by $X$. By the definition of the concavification operator and since $\underline{U}$ is continuous, we have

$$
\mathbb{E}\left[\underline{u}\left(X_{s}\right)\right]=\mathbb{E}\left[\underline{U}\left(\llbracket X_{1} \mid \mathcal{F}_{s} \rrbracket\right)\right] \leq \mathbb{E}\left[\operatorname{Cav}(\underline{U})\left(\llbracket X_{1} \mid \mathcal{F}_{s} \rrbracket\right)\right] \leq \operatorname{Cav}(\underline{U})\left(\llbracket X_{1} \rrbracket\right) \leq \operatorname{Cav}(\underline{U})(\mu),
$$

where the second inequality follows from Jensen's inequality (Lemma 6.1) and the third from the fact that $\operatorname{Cav}(\underline{U})$, being the pointwise limit of nondecreasing functions for the convex order, is itself nondecreasing. Using Fubini's Theorem, the right-hand side of (3.5) is less or equal than $\operatorname{Cav}(\underline{U})(\mu)$. To prove the converse inequality, let $\nu \in \Delta\left(\Delta\left(S_{K}\right)\right)$ be a probability whose barycenter is $\mu$ and such that $\int_{\Delta\left(S_{K}\right)} \underline{U}(\omega) d \nu(\omega)=\operatorname{Cav}(\underline{U})(\mu)$ (which exists by compactness). Let $(S, Y) \in \Delta\left(S_{K}\right) \times S_{K}$ be a pair of random variables such that $\llbracket S \rrbracket=\nu$ and $\llbracket Y \mid S \rrbracket=S$. Define a martingale by the relation $X_{1}=Y$ and $X_{t}=\mathbb{E}[Y \mid S]$ for all $t \in[0,1)$. Then

$$
\mathbb{E}\left[\int_{0}^{1} \underline{u}\left(X_{s}\right) d s\right]=\int_{0}^{1} \mathbb{E}[\underline{u}(\mathbb{E}[Y \mid S])] d s=\int_{0}^{1} \mathbb{E}[\underline{U}(\llbracket Y \mid S \rrbracket)] d s=\operatorname{Cav}(\underline{U})(\mu) .
$$

For the second assertion, if the property is true, then $X$ is clearly a maximizer. If $X$ is a maximizer and this property is false, then we obtain a contradiction using the inequalities 3.6 and integrating with respect to $s$ since the function $s \rightarrow \mathbb{E}\left[\underline{u}\left(X_{s}\right)\right]$ is càdlàg.

This result allows us to deduce the convergence of maximizers of the maximal variation problem to the maximizers of the continuous-time problem we just defined. This characterizes, using Proposition 3.1, the asymptotic behavior of the optimal strategies of the informed player in $\Gamma_{n}(\mu)$. We need the following notation. 
Notation 3.2. Given a discrete-time process $\left(L_{1}, . ., L_{n}\right)$, the continuous-time version of this process is defined by

$$
\Pi_{t}^{n}:=L_{\lfloor n t\rfloor} \quad \text { for } t \in[0,1],
$$

where $\lfloor a\rfloor$ denotes the greatest integer less or equal to a.

Proposition 3.3. Let $\left(L^{n}\right)$ be an asymptotically maximizing sequence of $\bar{\Psi}_{n}(\mu)$ in $\mathcal{M}_{n}(\mu)$, i.e. such that

$$
\Psi_{n}\left(L^{n}\right) \underset{n \rightarrow \infty}{\longrightarrow} \operatorname{Cav}(\underline{U})(\mu) .
$$

Then the continuous-time versions of these martingales define a weakly relatively compact sequence of laws for the Meyer-Zheng topology (see [27]) and any limit point belongs to

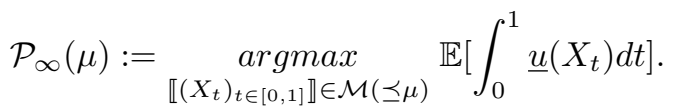

Proof. We refer to [27] for properties of the Meyer-Zheng topology on the Skorokhod space. This topology is the convergence in distribution over the set $\Delta\left(\mathbb{D}\left([0,1], S_{K}\right)\right)$, when the set $\mathbb{D}\left([0,1], S_{K}\right)$ of càdlàg functions is endowed with the topology of convergence in measure with respect to Lebesgue's measure (denoted $\boldsymbol{\lambda}$ ) together convergence of the value at time 1 : a sequence $y_{n}$ converges to $y$ if

$$
\forall \varepsilon>0, \lambda\left(\left\{\left|y_{n}(x)-y(x)\right| \geq \varepsilon\right\}\right) \underset{n \rightarrow \infty}{\longrightarrow} 0, \quad \text { and } \quad y_{n}(1) \underset{n \rightarrow \infty}{\longrightarrow} y(1)
$$

The subset $\mathcal{M}(\preceq \mu)$ of laws of martingales (in $\Delta\left(\mathbb{D}\left([0,1], S_{K}\right)\right)$ is weakly compact using Theorem 2 in $[27]$, the fact that the projection $\left(X_{t}\right)_{t \in[0,1]} \rightarrow X_{1}$ at time 1 is continuous on $\mathbb{D}\left([0,1], S_{K}\right)$ and that condition $\llbracket X_{1} \rrbracket \preceq \mu$ is weakly closed. Moreover, the functional $\llbracket\left(X_{t}\right)_{t \in[0,1] \rrbracket} \rightarrow \mathbb{E}\left[\int_{0}^{1} \underline{u}\left(X_{t}\right) d t\right]$ is weakly continuous. Let us denote $\Pi^{n}$ denote the sequence of continuous-time versions of $L^{n}$, and notice that $\llbracket \Pi^{n} \rrbracket \in \mathcal{M}(\preceq \mu)$ by construction. Assume, up to the extraction of some subsequence, that $\llbracket \Pi^{n} \rrbracket$ converges weakly to some limit $\llbracket \Pi \rrbracket$. Then, using the previous Propositions:

$$
\begin{aligned}
\Psi_{n}\left(\left(L_{q}\right)_{q=1, \ldots, n}\right) & \leq \mathbb{E}\left[\sum_{q=1}^{n} \frac{1}{n} \underline{U}\left(\llbracket L_{q} \mid L_{1}, . ., L_{q-1} \rrbracket\right)\right]+\frac{1}{n} C_{A} \mathbb{E}\left[\sum_{q=1}^{n}\left|L_{q}-L_{q-1}\right|\right] \\
& =\mathbb{E}\left[\sum_{q=1}^{n} \frac{1}{n} \underline{u}\left(L_{q-1}\right)\right]+\frac{1}{n} C_{A} \mathbb{E}\left[\sum_{q=1}^{n}\left|L_{q}-L_{q-1}\right|\right] \\
& \leq \mathbb{E}\left[\int_{0}^{1} \underline{u}\left(\Pi_{t}^{n}\right) d t\right]+\frac{1}{n} C_{A} \sqrt{K-1} \sqrt{n} \\
& \underset{n \rightarrow \infty}{\longrightarrow}\left[\int_{0}^{1} \underline{u}\left(\Pi_{t}\right) d t\right] .
\end{aligned}
$$

We conclude that $\llbracket \Pi \rrbracket \in \mathcal{P}_{\infty}(\mu)$ since by assumption the left-hand side of the above inequality converges to the value of problem (3.5).

Finally, discretizations of optimal limit martingales induce asymptotically optimal strategies.

Proposition 3.4. For all martingale $X$ in $\mathcal{P}_{\infty}(\mu)$ and all $n$, we have

$$
\Psi_{n}\left(\left(X_{\frac{q}{n}}\right)_{q=1, \ldots, n}\right) \geq \bar{\Psi}_{n}(\mu)-2 \frac{C_{A} \sqrt{K-1}}{\sqrt{n}}
$$

Proof. Let $X$ be a martingale such that $\llbracket\left(X_{t}\right)_{t \in[0,1]} \rrbracket \in \mathcal{P}_{\infty}(\mu)$.

$$
\begin{aligned}
n \Psi_{n}\left(\left(X_{\frac{q}{n}}\right)_{q=1, . ., n}\right) & \geq \mathbb{E}\left[\sum_{q=1}^{n} \underline{U}\left(\llbracket X_{\frac{q}{n}} \mid X_{\frac{1}{n}}, . ., X_{\frac{q-1}{n}} \rrbracket\right)\right]=\mathbb{E}\left[\sum_{q=1}^{n} \underline{u}\left(X_{\frac{q-1}{n}}\right)\right] \\
& \geq n \mathbb{E}\left[\int_{0}^{1} \underline{u}\left(X_{t}\right) d t\right]-n C_{A} \int_{0}^{1 / n} \mathbb{E}\left[\sum_{q=1}^{n}\left|X_{\frac{(q-1)}{n}+t}-X_{\underline{(q-1)}}\right|\right] d t \\
& \geq n \mathbb{E}\left[\int_{0}^{1} \underline{u}\left(X_{t}\right) d t\right]-n C_{A} \int_{0}^{1 / n} \mathbb{E}\left[\sum_{q=1}^{n}\left|X_{\frac{q}{n}}-X_{\underline{(q-1)}}\right|\right] d t \\
& =n \mathbb{E}\left[\int_{0}^{1} \underline{u}\left(X_{t}\right) d t\right]-C_{A} \mathbb{E}\left[\sum_{q=1}^{n}\left|X_{\frac{q}{n}}-X_{\frac{(q-1)}{n}}\right|\right] \\
& \geq n \mathbb{E}\left[\int_{0}^{1} \underline{u}\left(X_{t}\right) d t\right]-C_{A} \sqrt{K-1} \sqrt{n} \\
& \geq n \bar{\Psi}_{n}(\mu)-2 C_{A} \sqrt{K-1} \sqrt{n},
\end{aligned}
$$

where the third line follows from Jensen's Inequality. 
Using Remark 3.1, the discretization on the uniform partition of size $\frac{1}{n}$ of $[0,1]$ of any martingale in $\mathcal{P}_{\infty}(\mu)$ induces for all $n$ and $\varepsilon>0$ a strategy for P1 which is $2 C_{A} \frac{\sqrt{K-1}}{\sqrt{n}}+\varepsilon$-optimal in $\Gamma_{n}(\mu)$.

Remark 3.3. It follows from the characterization given in Proposition 3.2 that the maximizers of the continuoustime problem are degenerate in the following sense. If we have $\underline{U}(\mu)<\operatorname{Cav}(\underline{U})(\mu)$, then any optimal martingale jumps at time 0 to a position where $\left.\mathbb{E}\left[\underline{u}\left(X_{0}\right)\right]\right)=\operatorname{Cav}(\underline{U})(\mu)$ and then can either stop moving or evolve freely within a set on which $\underline{U}$ is linear. For the extremal situations, if $\underline{U}$ is strictly concave at $\mu$, then the optimal martingale never moves and is uniquely determined. On the contrary, if $\underline{U}$ is linear on $\Delta\left(S_{K}\right)$, then any martingale will be optimal. It means that the asymptotic behavior of the optimal strategies of P1 is in general degenerate from the dynamic point of view. The two preceding proofs can however be easily extended to time-dependent payoff functions (see section 5), in which case this degeneracy phenomenon disappears as shown in [11].

A Martingale version of the recurrence formula. The following result follows from the functional properties of $\Psi_{n}$ given in the appendix. Note that it provides another expression of the recurrence formula (and of the associated Shapley operator) for finite games.

Proposition 3.5. For any game $\Gamma_{n}(\mu)$ with $\mu \in \Delta\left(S_{K}\right)$ and $q=1, . ., n$, the following dynamic programming equation holds.

$$
n \underline{V}_{n}(\mu)=\sup _{\llbracket X_{1}, X_{2} \rrbracket \in \mathcal{M}_{2}(\mu)}\left(q \underline{V}_{q}\left(\llbracket X_{1} \rrbracket\right)+\mathbb{E}\left[(n-q) \underline{V}_{n-q}\left(\llbracket X_{2} \mid X_{1} \rrbracket\right)\right]\right)
$$

Proof. At first, $\Psi_{n}$ can be seen as a function on the set of martingale distributions $\mathcal{M}_{n}\left(S_{K}\right)=\underset{\mu \in \Delta\left(S_{K}\right)}{\cup} \mathcal{M}_{n}(\mu)$. The first inequality follows directly from Proposition 3.1. For any martingale $\llbracket L_{1}, . ., L_{n} \rrbracket$ in $\mathcal{M}_{n}(\mu), \llbracket L_{q}, L_{n} \rrbracket \in$ $\mathcal{M}_{2}(\mu)$ and $\llbracket L_{q+1}, \ldots, L_{n} \mid L_{q} \rrbracket$ is almost surely a martingale distribution. Therefore

$$
\begin{aligned}
n \Psi_{n}\left(\left(L_{k}\right)_{k=1, . ., n}\right) & =q \Psi_{q}\left(\left(L_{k}\right)_{k=1, . ., q}\right)+\mathbb{E}\left[(n-q) \Psi_{n-q}\left(\llbracket L_{q+1}, \ldots, L_{n} \mid L_{1}, . ., L_{q} \rrbracket\right)\right] \\
& \leq q \Psi_{q}\left(\left(L_{k}\right)_{k=1, \ldots, q}\right)+\mathbb{E}\left[(n-q) \bar{\Psi}_{n-q}\left(\llbracket L_{n} \mid L_{q} \rrbracket\right)\right] \\
& \leq q \bar{\Psi}_{q}\left(\llbracket L_{q} \rrbracket\right)+\mathbb{E}\left[(n-q) \bar{\Psi}_{n-q}\left(\llbracket L_{n} \mid L_{q} \rrbracket\right)\right] .
\end{aligned}
$$

The reverse inequality follows from the properties of $\Psi_{q}$. Using Lemma 6.2, the application

$$
\llbracket L_{1}, . ., L_{q} \rrbracket \rightarrow \Psi_{q}\left(L_{1}, . ., L_{q}\right)
$$

is concave and weakly upper semi-continuous on the compact set of martingale distributions. The graph of the mapping $\mu \rightarrow \mathcal{M}_{q}(\mu)$ being closed, there exists an optimal measurable selection $F$ from $\mu \in \Delta\left(S_{K}\right)$ to $\mathcal{M}_{q}(\mu)$ (see e.g. Proposition 7.33 in [6]) such that

$$
\Psi_{q}(F(\mu)) \geq \bar{\Psi}_{q}(\mu)
$$

The proof follows then easily. Take an optimal martingale $\llbracket X_{1}, X_{2} \rrbracket \in \mathcal{M}_{2}(\mu)$ in the right-hand side of (3.7). Let $g(x)$ denote a version of the conditional law of $X_{2}$ given $X_{1}$. There exists a martingale $\left(L_{1}, . ., L_{q}\right)$ such that $\llbracket L_{q} \rrbracket=\llbracket X_{1} \rrbracket$ and $\Psi_{q}\left(L_{1}, . ., L_{q}\right)=\bar{\Psi}_{q}\left(\llbracket L_{q} \rrbracket\right)$. We can construct a sequence of variables $\left(L_{q+1}, . ., L_{n}\right)$ whose conditional law given $\left(L_{1}, . ., L_{q}\right)$ is $F\left(g\left(L_{q}\right)\right)$. The construction implies therefore

$$
\begin{aligned}
n \Psi_{n}\left(L_{1}, . ., L_{n}\right) & =q \Psi_{q}\left(L_{1}, . ., L_{q}\right)+\mathbb{E}\left[(n-q) \Psi_{n-q}\left(\llbracket L_{q+1}, \ldots, L_{n} \mid L_{q} \rrbracket\right)\right] \\
& \geq q \underline{V}_{q}\left(\llbracket L_{q} \rrbracket\right)+\mathbb{E}\left[(n-q) \underline{V}_{n-q}\left(\llbracket L_{n} \mid L_{q} \rrbracket\right)\right],
\end{aligned}
$$

which concludes the proof.

\section{Dual game}

We introduce in this section an auxiliary game of complete information known in the finite case as the dual game, and that was at first introduced in De Meyer [18]. We show that the results obtained in Laraki [25] can be generalized in our model, including the representation of the concavification of $\bar{U}$ as the solution of a dual Hamilton-Jacobi equation. We deduce from this result a proof based on duality of the "Cav(u)" Theorem for the upper value functions of $\Gamma_{n}(\mu)$.

The model. We consider the game $\Gamma_{n}(\mu)$ defined by $\left(I, J, S_{K}, A\right)$ where $\mu \in \Delta\left(S_{K}\right)$. Given $\phi$ in the set of continuous functions $C\left(S_{K}\right)$, the dual game $\Gamma_{n}^{*}(\phi)$ is defined as follows. A strategy for P1 is a pair $(\mu, \sigma)$ where $\mu \in \Delta\left(S_{K}\right)$ and $\sigma \in \Sigma_{n}$. A strategy for P2 is some $\tau \in \mathcal{T}_{n}$. The payoff function is defined by

$$
g_{n}^{*}(\phi, \mu, \sigma, \tau):=\mathbb{E}_{\Pi(\mu, \sigma, \tau)}\left[\left\langle L, \frac{1}{n} \sum_{q=1}^{n} A\left(i_{q}, j_{q}\right)\right\rangle-\phi(L)\right]=g_{n}(\mu, \sigma, \tau)-\langle\phi, \mu\rangle .
$$

where $\langle\phi, \mu\rangle=\int_{S_{K}} \phi d \mu$. Let us now define the lower and upper value functions of this game as

$$
\underline{W}_{n}(\phi):=\sup _{(\mu, \sigma) \in \Delta\left(S_{K}\right) \times \Sigma_{n}} \inf _{\tau \in \mathcal{T}_{n}} g_{n}^{*}(\phi, \mu, \sigma, \tau),
$$




$$
\bar{W}_{n}(\phi):=\inf _{\tau \in \mathcal{T}_{n}} \sup _{(\mu, \sigma) \in \Delta\left(S_{K}\right) \times \Sigma_{n}} g_{n}^{*}(\phi, \mu, \sigma, \tau) .
$$

Our aim is to study the asymptotic behavior of the dual game, and precisely of $\bar{W}_{n}$.

Remark 4.1. In the following, the convex conjugation denoted by $*$ applied to functions defined on $\Delta\left(S_{K}\right)$ or $C\left(S_{K}\right)$ refers to the duality between the space $M\left(S_{K}\right)$ of signed Radon measures on $S_{K}$ and $C\left(S_{K}\right)$. All the functions defined on $\Delta\left(S_{K}\right)$ are extended on the whole space $M\left(S_{K}\right)$ by the value $+\infty$. Since $\Delta\left(S_{K}\right)$ is a weakly closed set in $M\left(S_{K}\right)$, if $F$ is convex continuous on $\Delta\left(S_{K}\right)$, its extension is l.s.c. and convex and therefore $F^{* *}=F$.

Proposition 4.1. For all $\phi \in C\left(S_{K}\right)$ and $\mu \in \Delta\left(S_{K}\right)$

$$
\begin{aligned}
& \underline{W}_{n}(\phi)=\left(-\underline{V}_{n}\right)^{*}(-\phi) \quad \text { and } \quad \underline{V}_{n}(\mu)=-\underline{W}_{n}^{*}(-\mu) \text {. } \\
& \bar{W}_{n}(\phi)=\left(-\bar{V}_{n}\right)^{*}(-\phi) \quad \text { and } \quad \bar{V}_{n}(\mu)=-\bar{W}_{n}^{*}(-\mu) .
\end{aligned}
$$

Proof. The first part follows directly from the definition and the preceding Remark. For the second, we have to apply a minmax Theorem. Indeed we have by definition

$$
\left(-\bar{V}_{n}\right)^{*}(-\phi)=\sup _{\mu \in \Delta\left(S_{K}\right)} \inf _{\tau \in \mathcal{T}_{n}} \sup _{\sigma \in \Sigma_{n}} g_{n}(\mu, \sigma, \tau)-\langle\phi, \mu\rangle
$$

and the result follows if we can invert the first sup and inf in this expression. In this context, we can apply the convex-like version of the minmax Theorem (Fan [22]). The argument is standard, the set $\mathcal{T}_{n}$ is not convex, but any convex combination of its elements is a general strategy and is equivalent by Kuhn's Theorem (see $[3])$ to a behavioral strategy which proves the convex-like hypothesis. On the other hand, $\Delta\left(S_{K}\right)$ is compact convex and the function we consider is concave and continuous with respect to $\mu$ using the same arguments as for Proposition 2.1.

Remark 4.2. As before, any function $\phi \in C\left(S_{K}\right)$ is implicitly identified with its extension on $\mathbb{R}^{K}$ which is equal to $\phi$ on $S_{K}$ and to $+\infty$ outside $S_{K}$. Its convex conjugate $\phi^{*}$ is therefore the usual convex conjugate of the extended function and if $\phi$ is convex on $S_{K}$, then $\phi=\phi^{* *}$.

Lemma 4.1. For all $\phi \in C\left(S_{K}\right)$,

$$
\bar{W}_{n}(\phi)=\inf _{\tau \in \mathcal{T}_{n}^{R}} \sup _{\left(i_{1}, . ., i_{n}\right) \in I^{n}} \phi^{*}\left(\frac{1}{n} \sum_{q=1}^{n} A\left(i_{q}, \tau_{q}\right)\right),
$$

where $A\left(i_{q}, \tau_{q}\right):=\int_{J} A\left(i_{q}, j_{q}\right) d \tau_{q}\left(i_{1}, . ., i_{q-1}\right)\left(j_{q}\right)$.

Proof. We deduce from Proposition 2.2 that

$$
\bar{W}_{n}(\phi) \leq \inf _{\tau \in \mathcal{T}_{n}^{R}} \sup _{(\mu, \sigma) \in \Delta\left(S_{K}\right) \times \Sigma_{n}} g_{n}^{*}(\phi, \mu, \sigma, \tau)=\inf _{\tau \in \mathcal{T}_{n}^{R}} \sup _{(\mu, \sigma) \in \Delta\left(S_{K}\right) \times \Sigma_{n}^{R}} g_{n}^{*}(\phi, \mu, \sigma, \tau)
$$

since $\mathcal{T}_{n}^{R} \subset \mathcal{T}_{n}$ and P1 cannot obtain a better payoff against a reduced strategy of P2 by using non-reduced strategies. The first inequality is an equality since from Proposition 2.2, P2 can replace any non-reduced strategy by a reduced strategy giving the same payoff against all reduced strategies of P1, i.e.

$$
\inf _{\tau \in \mathcal{T}_{n}^{R}} \sup _{(\mu, \sigma) \in \Delta\left(S_{K}\right) \times \Sigma_{n}^{R}} g_{n}^{*}(\phi, \mu, \sigma, \tau)=\inf _{\tau \in \mathcal{T}_{n}} \sup _{(\mu, \sigma) \in \Delta\left(S_{K}\right) \times \Sigma_{n}^{R}} g_{n}^{*}(\phi, \mu, \sigma, \tau) \leq \bar{W}_{n}(\phi) .
$$

The set of strategies $(\mu, \sigma) \in \Delta\left(S_{K}\right) \times \Sigma_{n}^{R}$ can be identified to the set of joint distributions on $S_{K} \times I^{n}$. Moreover, the structure of reduced strategies allows to integrate at first the payoff function with respect to $\tau$ conditionally on $\left(i_{1}, . ., i_{n}\right)$ and by Fubini Theorem

$$
\inf _{\tau \in \mathcal{T}_{n}^{R}} \sup _{(\mu, \sigma) \in \Delta\left(S_{K}\right) \times \Sigma_{n}^{R}} g_{n}^{*}(\phi, \mu, \sigma, \tau)=\inf _{\tau \in \mathcal{T}_{n}^{R}} \sup _{\pi \in \Delta\left(S_{K} \times I^{n}\right)} \mathbb{E}_{\pi}\left[\left\langle L, \frac{1}{n} \sum_{k=1}^{n} A\left(i_{k}, \tau_{k}\right)\right\rangle-\phi(L)\right] .
$$

The supremum over $\Delta\left(S_{K} \times I^{n}\right)$ is then equal to the supremum over $S_{K} \times I^{n}$. The supremum over $S_{K}$ being by definition $\phi^{*}\left(\frac{1}{n} \sum_{k=1}^{n} A\left(i_{k}, \tau_{k}\right)\right)$, this concludes the proof.

A recurrence formula. Let $C v\left(\mathbb{R}^{K}\right)$ denote the set of continuous convex functions $f$ on $\mathbb{R}^{K}$. We define the following family of operators $R_{\delta}$ on the set $C v\left(\mathbb{R}^{K}\right)$.

$$
\forall \delta \geq 0, \quad R_{\delta}(f)(x):=\inf _{\tau \in \Delta(J)} \sup _{i \in I} f(x+\delta A(i, \tau)) .
$$

Lemma 4.2. For all $\delta \geq 0$, the operator $R_{\delta}$ defines a map from $C v\left(\mathbb{R}^{K}\right)$ to itself. Moreover, for all $\varepsilon>0$, there exists a Borel measurable function $\tau_{\varepsilon}: \mathbb{R}^{K} \rightarrow \Delta(J)$ which is $\varepsilon$-optimal in the sense

$$
\forall x \in \mathbb{R}^{K}, \sup _{i \in I} f\left(x+\delta A\left(i, \tau_{\varepsilon}(x)\right)\right) \leq R_{\delta}(f)(x)+\varepsilon .
$$


Proof. $R_{\delta}(f)$ is real-valued since $A$ is bounded and $f$ continuous. For the convexity of $R_{\delta}(f)$ let $x_{1}, x_{2} \in \mathbb{R}^{K}$ and $\lambda \in[0,1]$. Let $\tau_{1}, \tau_{2}$ be $\eta$-optimal for the problems $R_{\delta}(f)\left(x_{1}\right)$ and $R_{\delta}(f)\left(x_{2}\right)$, then

$$
f\left(\lambda x_{1}+(1-\lambda) x_{2}+\delta A\left(i, \lambda \tau_{1}+(1-\lambda) \tau_{2}\right)\right) \leq \lambda R_{\delta}\left(x_{1}\right)+(1-\lambda) R_{\delta}\left(x_{2}\right)+\eta
$$

using the linearity of the integral and the convexity of $f$. The conclusion follows by sending $\eta$ to zero. For the functions $\tau_{\varepsilon}$, we proceed by discretization. For any fixed $\tau$, the function

$$
x \rightarrow \sup _{i \in I} f(x+\delta A(i, \tau))
$$

is continuous since it is locally bounded and convex. Since so is $R_{\delta}(f)$, any $\varepsilon / 2$ optimal $\tau$ in $x$ is therefore $\varepsilon$-optimal in a neighborhood of $x$. It follows that there exists a countable measurable partition of $\mathbb{R}^{K}$ and a function $\tau_{\varepsilon}$ constant on the element of the partition having the required properties.

We prove in the following Proposition that $\bar{W}_{n}$ satisfies a recurrence formula.

Proposition 4.2. For all $\phi \in C(P)$

$$
\bar{W}_{n}(\phi)=R_{1 / n}^{n}\left(\phi^{*}\right)(0), \quad \text { with } \quad R_{1 / n}^{n}=\underbrace{R_{1 / n} \circ \ldots \circ R_{1 / n}}_{n} .
$$

Proof. By induction, it is sufficient to prove that

$$
\bar{W}_{n}(\phi)=\inf _{\tau \in \mathcal{T}_{n-1}^{R}} \sup _{i_{1}, . ., i_{n-1}} R_{1 / n}\left(\phi^{*}\right)(x),
$$

with $x=\frac{1}{n} \sum_{q=1}^{n-1} A\left(i_{q}, \tau_{q}\right)$. For any reduced strategy $\tau \in \mathcal{T}_{n}^{R}$, we have the following inequality

$$
\begin{aligned}
\sup _{i_{1}, . ., i_{n}} \phi^{*}\left(\frac{1}{n} \sum_{q=1}^{n} A\left(i_{q}, \tau_{q}\right)\right) & =\sup _{i_{1}, . ., i_{n}} \phi^{*}\left(x+\frac{1}{n} A\left(i_{n}, \tau_{n}\left(i_{1}, . ., i_{n-1}\right)\right)\right) \\
& \geq \sup _{i_{1}, . ., i_{n-1}} R_{1 / n}\left(\phi^{*}\right)(x),
\end{aligned}
$$

which allows to prove a first inequality by taking the infimum over $\tau$ on both sides. For the reverse inequality, take $\tau_{n}=\tau_{\varepsilon}(x)$ with $\tau_{\varepsilon}$ given by the previous Lemma for $f=\phi^{*}$. For any $\left(\tau_{1}, . ., \tau_{n-1}\right) \in \mathcal{T}_{n-1}^{R}$, the above definition of $\tau_{n}$ defines a reduced strategy in $\mathcal{T}_{n}^{R}$ and we have

$$
\phi^{*}\left(\frac{1}{n} \sum_{q=1}^{n} A\left(i_{q}, \tau_{q}\right)\right)=\phi^{*}\left(x+\frac{1}{n} A\left(i_{n}, \tau_{\varepsilon}(x)\right)\right) \leq R_{1 / n}\left(\phi^{*}\right)(x)+\varepsilon,
$$

which allows easily to conclude by taking successively the supremum over $\left(i_{1}, . ., i_{n-1}\right)$ and the infimum over $\left(\tau_{1}, . . \tau_{n-1}\right)$ and then by sending $\varepsilon$ to zero.

The dual PDE formulation. In order to simplify the following statements, let us extend the function $\bar{u}$ to $\mathbb{R}^{K}$.

$$
\forall x \in \mathbb{R}^{K}, \bar{u}(x):=\inf _{\tau \in \Delta(J)} \sup _{\sigma \in \Delta(I)} \mathbb{E}_{\Pi\left(\delta_{x}, \sigma, \tau\right)}[\langle L, A(i, j)\rangle]=\inf _{\tau \in \Delta(J)} \sup _{\sigma \in \Delta(I)} \int_{I \times J}\langle x, A(i, j)\rangle d(\sigma \otimes \tau)(i, j) .
$$

The following properties are obvious and stated without proof.

Lemma 4.3. The above defined extension of $\bar{u}$ is positively homogenous and $C_{A}$-Lipschitz on $\mathbb{R}^{K}$.

Let $B U C\left(\mathbb{R}^{K}\right)$ denote the set of bounded uniformly continuous functions on $\mathbb{R}^{K}$ endowed with the uniform norm and for Lipschitz functions, let $\operatorname{Lip}(f)$ denote the Lipschitz constant of $f$.

Lemma 4.4. The family of operators $\left(R_{\delta}\right)_{\delta \geq 0}$ maps $B U C\left(\mathbb{R}^{K}\right)$ into itself and has the following properties

1) For all $f \in B U C\left(\mathbb{R}^{K}\right), R_{0}(f)=f$.

2) For all $f \in B U C\left(\mathbb{R}^{K}\right)$, the function $\delta \rightarrow R_{\delta}(f)$ is continuous .

3) There exists a constant $C_{1}$ such that $\left\|R_{\delta}(f)\right\|_{\infty} \leq C_{1} \delta+\|f\|_{\infty}$.

4) For all $f, g \in B U C\left(\mathbb{R}^{K}\right), \alpha \in \mathbb{R}, R_{\delta}(f+\alpha)=R_{\delta}(f)+\alpha$.

5) For all $f, g \in B U C\left(\mathbb{R}^{K}\right)$, $\left\|R_{\delta}(f)-R_{\delta}(g)\right\|_{\infty} \leq\|f-g\|_{\infty}$.

6) For all $f \in B U C\left(\mathbb{R}^{K}\right)$ which is Lipschitz, $\operatorname{Lip}\left(R_{\delta}(f)\right) \leq \operatorname{Lip}(f)$ and there exists a constant $C_{2}$ depending only on Lip $(f)$ such that $\left\|R_{\delta}(f)-f\right\|_{\infty} \leq C_{2} \delta$.

7) There exists a constant $C_{3}$ such that for all $f \in B U C\left(\mathbb{R}^{K}\right)$ of class $C^{2}$ with bounded derivatives, we have

$$
\left\|\frac{R_{\delta}(f)-f}{\delta}-\bar{u}(\nabla f)\right\|_{\infty} \leq C_{3} \delta\left(\left\|\nabla^{2} f\right\|_{\infty}\right) .
$$


Proof. The first six points are obvious. For the last one, we have using the second order Taylor expansion of $f$

$$
f(x+\delta A(i, \tau))=f(x)+\delta\langle\nabla f(x), A(i, \tau)\rangle+\delta^{2} R(x, i, \tau),
$$

where $|R(x, i, \tau)| \leq C\left\|\nabla^{2} f\right\|_{\infty}$ for some constant $C$ depending only on $A$. It follows that

$$
\begin{aligned}
& \left|\underset{\tau \in \Delta(J)}{\inf \sup _{i \in I} f(x+\delta A(i, \tau))-} \inf _{\tau \in \Delta(J)} \sup _{i \in I}\left[f(x)+\delta \int_{J}\langle\nabla f(x), A(i, j)\rangle d \tau(j)\right]\right| \\
& \leq C \delta^{2}\left\|\nabla^{2} f\right\|_{\infty},
\end{aligned}
$$

which concludes the proof since from the definition of $\bar{u}$

$$
\inf _{\tau \in \Delta(J)} \sup _{i \in I} \int_{J}\langle\nabla f(x), A(i, j)\rangle d \tau(j)=\bar{u}(\nabla f(x)) .
$$

These properties allow us to apply the results on approximation schemes of Souganidis [30] as in Laraki [25].

Proposition 4.3. For all convex Lipschitz functions $f$ on $\mathbb{R}^{K}$, define $f^{n}$ on $[0,1] \times \mathbb{R}^{K}$ by

$$
f^{n}(1, x)=f(x) \text { and } \quad f^{n}(t, x)=R_{\frac{q+1}{n}-t}\left(f^{n}\left(\frac{q+1}{n}, .\right)\right)(x) \text { for } t \in\left[\frac{q}{n}, \frac{q+1}{n}\right), q=0, . ., n-1
$$

Then, $f^{n}(t, x) \rightarrow \chi(t, x)$ where $\chi$ is the unique viscosity solution of the following Hamilton-Jacobi equation

$$
\left\{\begin{array}{cc}
\frac{\partial \chi}{\partial t}(t, x)+\bar{u}\left(\nabla_{x} \chi(t, x)\right)=0 & \text { for }(t, x) \in[0,1) \times \mathbb{R}^{K} \\
\chi(1, x)=f(x) & \text { for } x \in \mathbb{R}^{K}
\end{array}\right.
$$

in the class of uniformly continuous functions. Moreover, there exists a constant $C_{0}$ depending uniquely on the Lipschitz constant of $f$ such that

$$
\left|f^{n}(t, x)-\chi(t, x)\right| \leq \frac{C_{0}}{\sqrt{n}}
$$

Proof. That the solutions are unique within the class of uniformly continuous functions for the considered equation follows from Bardi and Evans [5] (Theorem 3.1), we always consider these solutions in the following. Note that if $\chi$ is the solution to (4.3) and $\alpha \in \mathbb{R}$, then $\chi+\alpha$ is the solution to the same equation with boundary condition $\chi(1,)=.f+\alpha$. Using property 4 in Lemma 4.4 , this allows to assume that $f(0)=0$. For all $n$, $f^{n}(t, x)$ depends only of the restriction of $f$ to the ball $B\left(x, C_{A}\right)$. Therefore $f$ can be replaced by a truncation $\beta_{b}(f)$ with $\beta_{b}$ defined by

$$
\beta_{b}: \mathbb{R} \rightarrow \mathbb{R}: x \rightarrow\left\{\begin{array}{ccc}
b & \text { if } & x \geq b \\
x & \text { if } & |x| \leq b \\
-b & \text { if } & x \leq-b
\end{array}\right.
$$

for some sufficiently large $b$. Lemmas 4.3 and 4.4 allow us to apply the introductory Theorem in [30] which implies that $f^{n}(t, x) \rightarrow \chi_{b}(t, x)$ where $\chi_{b}$ is the unique viscosity solution of the following Hamilton-Jacobi equation

$$
\left\{\begin{array}{cc}
\frac{\partial \chi}{\partial t}(t, x)+\bar{u}\left(\nabla_{x} \chi(t, x)\right)=0 & \text { for }(t, x) \in[0,1) \times \mathbb{R}^{K} \\
\chi(1, x)=\beta_{b}(f(x)) & \text { for } x \in \mathbb{R}^{K}
\end{array}\right.
$$

Moreover, the cited Theorem asserts that there exists a constant $C_{0}$ which depends only on $\left\|\beta_{b}(f)\right\|_{\infty}$ and $\operatorname{Lip}\left(\beta_{b}(f)\right)$ such that

$$
\left|f^{n}(t, x)-\chi_{b}(t, x)\right| \leq \frac{C_{0}}{\sqrt{n}}
$$

for $n$ large enough (the bound depending also only on the same constants (see [30] p.21)). Since these two quantities are bounded by $\left(C_{A} \vee 1\right) \operatorname{Lip}(f)$, this constant $C_{0}$ depends only on $\operatorname{Lip}(f)$ and is independent of $b$. Using that $\bar{u}$ is positively homogenous and Lipschitz, Proposition 6.1 in [1] shows that $\chi_{b}=\beta_{b}(\chi)$ where $\chi$ is the unique solution of (4.3) (with boundary condition $f$ ). We can therefore fix $b$ sufficiently large so that $\chi(t, x)=\chi_{b}(t, x)$ which concludes the proof.

Definition 4.1. For any function $\phi \in C\left(S_{K}\right)$, we define $W$ by $W(\phi)=\chi(0,0)$ where $\chi$ is the unique viscosity solution of the following Hamilton-Jacobi equation

$$
\left\{\begin{array}{cc}
\frac{\partial \chi}{\partial t}(t, x)+\bar{u}\left(\nabla_{x} \chi(t, x)\right)=0 & \text { for }(t, x) \in[0,1) \times \mathbb{R}^{K} \\
\chi(1, x)=\phi^{*}(x) & \text { for } x \in \mathbb{R}^{K}
\end{array}\right.
$$

in the class of uniformly continuous functions.

Gathering the preceding results, we obtain 
Corollary 4.1. There exists a constant $C_{0}$ such that for all $\phi \in C\left(S_{K}\right)$,

$$
\bar{W}_{n}(\phi) \leq W(\phi)+\frac{C_{0}}{\sqrt{n}} .
$$

Proof. Apply the preceding result to all the function $\phi^{*}$ for $\phi \in C\left(S_{K}\right)$. Since for $\phi \in C\left(S_{K}\right), \phi^{*}$ is 1-Lipschitz, the constant $C_{0}$ does not depend on $\phi$.

Let us now relate this result with the study of the primal game $\Gamma_{n}(\mu)$. For this, let us recall Hopf's formula, which gives an explicit expression for the solution of (4.5).

Proposition 4.4. For all $\phi \in C\left(S_{K}\right), W(\phi)=\left(\phi^{* *}-\bar{u}\right)^{*}(0)$.

Proof. The solution $\chi$ of (4.5) is given by the Hopf's formula (see Bardi-Evans [5] Theorem 3.1)

$$
\chi(1-t, x)=\sup _{p \in \mathbb{R}^{K}} \inf _{q \in \mathbb{R}^{d}}\left[\phi^{*}(q)+\langle p, x-q\rangle+t \bar{u}(p)\right] .
$$

And a direct computation shows that $\chi(0,0)=\left(\phi^{* *}-\bar{u}\right)^{*}(0)$.

The Hopf's solution is actually related to the concave conjugate of $\bar{U}$.

Lemma 4.5. The conjugate of the function $\bar{U}$ defined on the set $C\left(S_{K}\right)$ verifies

$$
\forall \phi \in C\left(S_{K}\right), \quad(-\bar{U})^{*}(-\phi)=W(\phi) .
$$

Proof. It follows from the definition that

$$
\begin{aligned}
(-\bar{U})^{*}(-\phi) & =\sup _{\mu \in \Delta\left(S_{K}\right)} \bar{U}(\mu)-\langle\phi, \mu\rangle \\
& =\sup _{x \in S_{K}}\left(\sup _{\mu: \mathbb{E}(\mu)=x} \bar{u}(x)-\langle\phi, \mu\rangle\right) \\
& =\sup _{x \in S_{K}} \bar{u}(x)-\phi^{* *}(x)=\left(\phi^{* *}-\bar{u}\right)^{*}(0)=W(\phi),
\end{aligned}
$$

where the third equality follows from the continuity of $\phi$ which implies

$$
\inf _{\mu: \mathbb{E}(\mu)=x}\langle\phi, \mu\rangle=\phi^{* *}(0) .
$$

We are now able to prove the dual version of the "Cav(u)" Theorem.

of Theorem 1.2. Note that the first inequality can be proved using the splitting method described in Proposition 2.8 p.188 in [26]. Indeed, by replacing $\varepsilon$-optimal strategy by $\varepsilon$-best reply to the next mixed action of P2 in the appropriate non-revealing game, we deduce that P1 can defend $\operatorname{Cav}(\bar{U})$ in $\Gamma_{n}(\mu)$ (and also in the infinitely repeated game). For the second inequality, we have

$$
\left(-\bar{V}_{n}\right)^{*}(-\phi)=\bar{W}_{n}(\phi)=R_{1 / n}^{n}\left(\phi^{*}\right)(0) \leq W(\phi)+C_{0} n^{-1 / 2} .
$$

Using that $(-\bar{U})^{*}(-\phi)=W(\phi)$, the preceding relation implies

$$
\bar{V}_{n}(\mu) \leq-(-\bar{U})^{* *}(\mu)+C_{0} n^{-1 / 2} .
$$

Since $\bar{U}$ is concave and weakly continuous, we have that $-(-\bar{U})^{* *}(\mu)=\operatorname{Cav}(\bar{U})$ and this concludes the proof.

In order to study the optimal strategies of P2, we need the following technical Lemma.

Lemma 4.6. For all $\mu \in \Delta\left(S_{K}\right)$,

$$
\exists \phi \in \partial \operatorname{Cav}(\bar{U})(\mu):=\left\{\psi \in C\left(S_{K}\right) \mid W(\psi)+\langle\psi, \mu\rangle=\operatorname{Cav}(\bar{U})(\mu)\right\} .
$$

Moreover, $\phi$ can be chosen convex and $C_{A}$-Lipschitz.

Proof. Since $\operatorname{Cav}(\bar{U})$ is concave and upper semi-continuous, we have

$$
\operatorname{Cav}(\bar{U})(\mu)=\inf _{\psi \in C\left(S_{K}\right)} W(\psi)+\langle\psi, \mu\rangle
$$

Since $W$ depends on $\psi$ only through the restriction of $\psi^{*}$ to the ball $B\left(0, C_{A}\right)$, one can replace $\psi$ by $\psi^{* *}$ which implies that the infimum can be taken over convex functions. Moreover, one can replace any convex function $\psi$ by its (Moreau-Yosida) approximation defined by

$$
\widetilde{\psi}(x)=\left\{\begin{array}{ll}
\inf _{y \in S_{K}} \psi(y)+C_{A}|y-x| & x \in S_{K} \\
+\infty & x \notin S_{K}
\end{array} .\right.
$$


Indeed, $\widetilde{\psi}$ is convex as a marginal function, $C_{A}$-Lipschitz and $\widetilde{\psi}^{*}$ coincides with $\psi^{*}$ on $B\left(0, C_{A}\right)$. Using that $W(\psi+\alpha)=W(\psi)-\alpha$, we can assume that $\psi\left(e_{1}\right)=0$, and the infimum is finally taken over the set of $C_{A^{-}}$ lipschitz convex functions vanishing in $e_{1}$. The function $W$ being 1-Lipschitz for the uniform norm (using point 5 of Lemma 4.4), existence of a minimum in this set follows from Ascoli's Theorem.

Finally, this dual formulation provides $\mathcal{O}(\sqrt{n})$-optimal strategies for the uninformed player.

Proposition 4.5. For all $\mu \in \Delta\left(S_{K}\right), \phi \in \partial \operatorname{Cav}(\bar{U})(\mu), \varepsilon>0$ and $n \in \mathbb{N}^{*}$, any $\varepsilon$-optimal strategy $\tau$ in $\Gamma_{n}^{*}(\phi)$ is $\varepsilon+C_{0} n^{-\frac{1}{2}}$ optimal in $\Gamma_{n}(\mu)$.

Proof. Existence of $\phi$ follows from the previous Lemma. By assumption, the strategy $\tau$ is such that

$$
\sup _{\sigma \in \Sigma_{n}} g_{n}(\mu, \sigma, \tau)-\langle\phi, \mu\rangle \leq \bar{W}_{n}(\phi)+\varepsilon \leq W(\phi)+C_{0} n^{-\frac{1}{2}}+\varepsilon
$$

and the result follows since by definition of $\partial \operatorname{Cav}(\bar{U})(\mu)$.

Remark 4.3. Using Theorem 3.1 p.191 in [26], P2 can guarantee lim $\bar{V}_{n}=\operatorname{Cav}(\bar{U})$ in the infinitely repeated game.

Let us now prove the last result announced in section 1 concerning the uniform value.

of Corollary 1.1. It results directly from Theorems 1.1 and 1.2 and Remarks 3.2 and 4.3 .

The results concerning the upper value functions and optimal strategies of P2 (and thus Corollary 1.1 which does not depend on the study of the lower value functions) can also be derived more directly. As for finite games (see Kohlberg [24]), one can construct from the supergradient vector $\phi$ given in Lemma 4.6 an $\varepsilon$-optimal strategy in the infinitely repeated game using Blackwell's approachability (see Blackwell [8]). A short proof is provided below for the sake of completeness.

Proposition 4.6. Given $\mu \in \Delta\left(S_{K}\right)$ and a convex function $\phi \in \partial \operatorname{Cav}(\bar{U})(\mu)$, the convex set

$$
C_{\varepsilon}=\left\{q \in \mathbb{R}^{K} \mid \forall x \in S_{K},\langle q, x\rangle \leq \phi(x)+\varepsilon\right\}
$$

is a $B$-set for $P 2$ in the game with vector payoffs $A(i, j)$ and action sets $I, J$. Any strategy $\tau_{\varepsilon}$ adapted to this set is $\varepsilon$-optimal in the infinitely repeated game $\Gamma_{\infty}(\mu)$.

Proof. Replacing $\phi$ by $\phi+W(\phi)$, we obtain

$$
\operatorname{Cav}(\bar{U})(\mu)=\langle\phi, \mu\rangle \text {, and } \forall \nu \in \Delta\left(S_{K}\right), \bar{U}(\nu) \leq \operatorname{Cav}(\bar{U})(\nu) \leq\langle\phi, \nu\rangle .
$$

$C_{\varepsilon}$ is convex closed and lower comprehensive. Moreover, since $\phi+\varepsilon$ is convex and continuous, it is the supremum of the affine functions below it, i.e.

$$
\forall x \in S_{K}, \sup _{q \in C_{\varepsilon}}\langle q, x\rangle=\phi(x)+\varepsilon .
$$

Let us prove that $C_{\varepsilon}$ is a B-set for P2 in the game with vector-payoffs $A(i, j) \in \mathbb{R}^{K}$. Using convexity, it is sufficient to prove that for all $z \in R^{K}$,

$$
\inf _{\tau \in \Delta(J)} \sup _{i \in I}\langle z, A(i, \tau)\rangle \leq \sup _{q \in C_{\varepsilon}}\langle q, z\rangle .
$$

The right-hand side being equal to $+\infty$ whenever $z \notin \mathbb{R}_{+}^{K}$, we can assume that $z \in \mathbb{R}_{+}^{K}$ and clearly that $z \neq 0$. By homogeneity, this reduces to the case $z \in S_{K}$, and we have to prove that

$$
\underset{\tau \in \Delta(J)}{\inf } \sup _{i \in I}\langle z, A(i, \tau)\rangle \leq \phi(z)+\varepsilon .
$$

This follows by construction since the left-hand side is equal to $\bar{U}\left(\delta_{z}\right) \leq \phi(z)$. Now, any strategy $\tau_{\varepsilon}$ for P2 adapted to the $B$-set $C_{\varepsilon}$ (existence follows using the same measurable selection result as for lemma 4.2) is such that (see [8] or Corollary 4.6 p.104 in [26])

$$
\forall \sigma \in \Sigma_{n}, \mathbb{E}_{\Pi\left(\mu, \sigma, \tau_{\varepsilon}\right)}\left[d\left(C_{\varepsilon}, \frac{1}{n} \sum_{q=1}^{n} A\left(i_{q}, j_{q}\right)\right)\right] \leq \frac{C_{A}}{\sqrt{n}} .
$$

Denoting by $u_{n}$ the projection of $\left.\frac{1}{n} \sum_{q=1}^{n} A\left(i_{q}, j_{q}\right)\right)$ on $C_{\epsilon}$ and $d_{n}$ its distance to $C_{\varepsilon}$, we deduce that $\forall \sigma \in \Sigma_{n}$

$$
\begin{aligned}
\mathbb{E}_{\Pi\left(\mu, \sigma, \tau_{\varepsilon}\right)}\left[\left\langle L, \frac{1}{n} \sum_{q=1}^{n} A\left(i_{q}, j_{q}\right)\right\rangle\right] & \leq \mathbb{E}[|L|] d_{n}+\mathbb{E}\left[\left\langle L, u_{n}\right\rangle\right] \\
& \leq \frac{C_{A}}{\sqrt{n}}+\mathbb{E}[\phi(L)]+\varepsilon=\operatorname{Cav}(\bar{U})(\mu)+\varepsilon+\frac{C_{A}}{\sqrt{n}}
\end{aligned}
$$

which ends the proof. 


\section{Extensions.}

General evaluations. Instead of considering the $n$-times repeated game, one may consider the $\lambda$-discounted game or more generally the infinitely repeated game where payoffs are evaluated as follows with obvious notations

$$
g_{\theta}(\mu, \sigma, \tau)=\mathbb{E}_{\Pi(\mu, \sigma, \tau)}\left[\left\langle L, \sum_{q=1}^{\infty} \theta_{q} A\left(i_{q}, j_{q}\right)\right\rangle\right] .
$$

for some $\theta \in \Delta\left(\mathbb{N}^{*}\right)$. Denoting $\underline{V}_{\theta}$ and $\bar{V}_{\theta}$ the associated lower and upper value functions, all our results extend for sequences $\theta^{n}$ such that $\varepsilon_{n}:=\sup _{q} \theta_{q}^{n} \rightarrow 0$. Precisely, we have

$$
\underline{V}_{\theta}(\mu)=\sup _{\llbracket\left(L_{q}\right)_{q \geq 1} \rrbracket \in \mathcal{M}_{\infty}(\mu)} \mathbb{E}\left[\sum_{q=1}^{+\infty} \theta_{q} \underline{V}_{1}\left(\llbracket L_{q} \mid L_{1}, . ., L_{q-1} \rrbracket\right)\right],
$$

where $\mathcal{M}_{\infty}(\mu)$ denotes the set of laws of $S_{K}$-valued martingales $\left(L_{q}\right)_{q \geq 1}$ such that the law of the almost sure limit $L_{\infty}$ of the martingale is dominated by $\mu$. Lemma 3.4 can be adapted to obtain

$$
\mathbb{E}\left[\sum_{q \geq 1} \theta_{q}\left|L_{q}-L_{q-1}\right|\right] \leq \sqrt{K-1} \sqrt{\varepsilon_{n}}
$$

for any martingale with values in $S_{K}$ (it follows from a direct application of Cauchy-Schwarz inequality). The analysis made in section 3 leads to

$$
\operatorname{Cav}(\underline{U})(\mu) \leq \underline{V}_{\theta^{n}}(\mu) \leq \operatorname{Cav}(\underline{U})(\mu)+C_{A} \sqrt{K-1} \sqrt{\varepsilon_{n}} .
$$

The posterior martingale is a process $\left(L_{q}\right)_{q \geq 1}$ with law in $\mathcal{M}_{\infty}(\mu)$, and the corresponding continuous-time martingale is piecewise constant on the partition of $[0,1]$ formed by the sequence $\left(t_{q}\right)_{q \geq 0}$ with $t_{0}=0$, and $t_{q}=\sum_{m=1}^{q} \theta_{m}$ for $q \geq 1$. For probabilities $\theta$ with infinite support, the value at time 1 of this continuous-time martingale can be defined as $L_{\infty}$. Propositions 3.3 and 3.4 can then be extended in an obvious way.

For the lower value functions, probabilities $\theta$ with infinite support can be approximated by $\theta^{N}$ defined by $\theta_{q}^{N}=\theta_{q}$ for $q \leq N-1$ and $\theta_{N}^{N}=\sum_{q \geq N} \theta_{q}$. The same method leads to

$$
\operatorname{Cav}(\bar{U})(\mu) \leq \bar{V}_{\theta^{n}}(\mu) \leq \operatorname{Cav}(\bar{U})(\mu)+C_{0} \sqrt{\varepsilon_{n}} .
$$

Time-dependent payoff. Consider that $A(t, i, j)$ is a bounded continuous function defined on $[0,1] \times I \times J$ which is uniformly Lipschitz-continuous of constant $C^{\prime}$ with respect to $t$. The $n$-times repeated game (or any general evaluation as above) with time-varying payoff function is then defined as $\Gamma_{n}(\mu)$, except that the payoff function is now

$$
g_{n}(\mu, \sigma, \tau):=\mathbb{E}_{\Pi(\mu, \sigma, \tau)}\left[\frac{1}{n}\left\langle L, \sum_{q=1}^{n} A\left(\frac{q-1}{n}, i_{q}, j_{q}\right)\right\rangle\right] .
$$

Define for all $t \in[0,1]$ the lower value functions $\underline{V}_{1}(t,),. \underline{U}(t,$.$) and \underline{u}(t,$.$) by replacing the payoff by A(t,$.$) in$ the previous definitions and note that they are also uniformly Lipschitz with respect to $t$. The same analysis leads to

$$
-\frac{C_{A} \sqrt{K-1}}{\sqrt{n}}-\frac{C^{\prime}}{2 n} \leq \underline{V}_{n}(\mu)-\sup _{\llbracket\left(X_{t}\right)_{t \in[0,1]} \rrbracket \in \mathcal{M}(\preceq \mu)} \mathbb{E}\left[\int_{0}^{1} \underline{u}\left(t, X_{t}\right) d t\right] \leq \frac{C_{A} \sqrt{K-1}}{\sqrt{n}} .
$$

The first inequality follows from the proof of Proposition 3.4, where we have an additional error term since

$$
\sum_{q=1}^{n} \underline{u}\left(\frac{q-1}{n}, X_{\frac{q-1}{n}}\right) \geq n \int_{0}^{1} \underline{u}\left(t, X_{t}\right) d t-n C_{A} \int_{0}^{1 / n} \sum_{q=1}^{n}\left|X_{\frac{q-1}{n}}-X_{\frac{q-1}{n}+t}\right|-C^{\prime} n^{2} \int_{0}^{1 / n} t d t .
$$

The second inequality is proved as in the second part of the proof of Theorem 1.1, using that the supremum over piecewise-constant martingales is lower or equal than the supremum over all martingales. Propositions 3.3 and 3.4 can also be extended in an obvious way.

For the upper value functions, define the time-dependent versions $\bar{U}(t,$.$) and \bar{u}(t,$.$) as above. Let also \bar{W}_{n}(\phi)$ denote the upper value of the corresponding dual game. The results on viscosity solutions used in section 4 still hold in this case (namely Theorems 2.1 in [30] and Proposition 6.1 in [1] ). Replacing the family of operators defined before Lemma 4.2 by the corresponding time-dependent versions,

$$
R_{t, \delta}(f)=\inf _{\tau \in \Delta(J)} \sup _{i \in I} f\left(x+\delta \int_{J} A(t, i, j) d \tau(j)\right)
$$

the same analysis leads to

$$
\left|\bar{W}_{n}(\phi)-W(\phi)\right| \leq \frac{C_{0}}{\sqrt{n}}
$$


where $W(\phi)=\chi(0,0)$ and $\chi$ denotes the unique viscosity solution of the following Hamilton-Jacobi equation

$$
\left\{\begin{array}{cc}
\frac{\partial \chi}{\partial t}(t, x)+\bar{u}\left(t, \nabla_{x} \chi(t, x)\right)=0 & \text { for }(t, x) \in(0,1] \times \mathbb{R}^{K} \\
\chi(1, x)=\phi^{*}(x) & \text { for } x \in \mathbb{R}^{K}
\end{array}\right.
$$

in the class of uniformly continuous functions.

Note that using Lemma 4.2 (together with Theorem 2.1 in [30]), $x \rightarrow \chi(t, x)$ is convex for all $t \in[0,1]$. However, Lemma 4.5, and the link with Hopf formula (Proposition 4.4) do not extend to this new context. These results can be replaced by Lemma 6.4 (proved in the appendix) which relies on the notion of dual solutions developed in [12] and on Proposition 4.1 in [11]. This leads directly to the following version of (5.1), using the same method as for Theorem 1.2:

$$
\left|\bar{V}_{n}(\mu)-\sup _{\llbracket\left(X_{t}\right)_{t \in[0,1]} \rrbracket \in \mathcal{M}(\preceq \mu)} \mathbb{E}\left[\int_{0}^{1} \bar{u}\left(t, X_{t}\right) d t\right]\right| \leq \frac{C_{0}}{\sqrt{n}} .
$$

Compact metric state space. One may consider a continuum of states as in [13]. Let $K$ be a compact metric space and assume that the payoff function $A(k, i, j)$ defined on $K \times I \times J$ is uniformly Lipschitz with respect to $k$ (one may also add a dependence in time). A similar analysis can certainly be made in this context, at least for the lower value functions, but would require however to extend Lemma 3.4 for measure-valued martingales.

More general information structure. The main idea of Proposition 3.1 is that P1 controls the posterior beliefs martingale. For any martingale (or revelation process), he can maximize his expected payoff under the constraint that he will not reveal more than prescribed by the chosen process. This argument seems to be meaningful in any extensive form zero-sum game in which P1 controls the posterior beliefs of P2 on some state variable. Further works will be done in this direction for models in which (for example) P1 receives a sequence of signals during the game drawn according to an exogenous joint distribution depending on the state variable.

\section{Appendix.}

Definition 6.1. The Wasserstein distance of order 1 is defined on the set $\Delta^{1}\left(\mathbb{R}^{K}\right)$ of probabilities with finite moment of order 1 by

$$
d_{W}(\mu, \nu)=\min _{\pi \in \mathcal{P}(\mu, \nu)}\left(\int|y-x| d \pi(x, y)\right)=\min \left\{\|X-Y\|_{L^{1}} \mid X \sim \mu, Y \sim \nu\right\}
$$

where $|$.$| is the usual euclidian norm on \mathbb{R}^{K}$.

The following Theorem is well-known and allows to construct variables with prescribed conditional laws.

Theorem 6.1. (Blackwell-Dubins [9])

Let $E$ be a polish space with $\Delta(E)$ the set of Borel probabilities on $E$, and $([0,1], \mathcal{B}([0,1]), \lambda)$ the unit interval equipped with Lebesgue's measure. There exists a measurable mapping

$$
\Phi: \Delta(E) \times[0,1] \longrightarrow E
$$

such that for all $\mu \in \Delta(E)$, the law of $\Phi(\mu, U)$ is $\mu$ where $U$ is the canonical element in $[0,1]$.

\subsection{Technical proofs.}

Proof of Lemma 3.2. At first, this statement is equivalent to the existence of a measurable function

$$
H_{\varepsilon}: S_{K} \times \Delta\left(S_{K}\right) \rightarrow \Delta(I)
$$

having the property that the strategy $L \rightarrow H_{\varepsilon}(L, \mu)$ is $\varepsilon$-optimal in $\Gamma_{1}(\mu)$ using the correspondence $\phi_{\varepsilon}(L, \mu, U)=$ $\Phi\left(H_{\varepsilon}(L, \mu), U\right)$ where $\Phi$ is given by Theorem 6.1 . The main problem here is that there is no easily tractable topology on the set of $\mathrm{P} 1$ strategies (usual Young topologies require a reference measure $\mu$ which will be a variable for us), but thanks to the regularity of the payoff function with respect to the state variable, we can proceed by a standard discretization method. Let us fix some $0<\eta<1$. Let $\left(\Omega_{q}\right)_{q=1, . ., Q}$ be a finite measurable partition of $S_{K}$ of mesh smaller than $\eta$ and $\left(x_{q}\right)_{q=1, . . Q}$ a sequence of points such that $x_{q} \in \Omega_{q}$ for all $q$. Define then $\Lambda$ as the subset of convex combinations $\lambda \in \Delta(Q)$ such that for all $q=1, . ., Q, \lambda_{q} \in\left\{\frac{k}{N}, k=0, . ., N\right\}$ for some $N$ to be fixed later. Let us define $\lambda$ as the discretization on this grid.

$$
\lambda: \Delta\left(S_{K}\right) \rightarrow \Lambda: \mu \rightarrow\left\{\begin{array}{c}
\lambda_{q}(\mu)=\frac{\left\lfloor\mu\left(\Omega_{q}\right) N\right\rfloor}{N} \quad \text { for } \quad q=1, \ldots, Q-1 \\
\lambda_{Q}(\mu)=1-\sum_{q=1}^{Q-1} \lambda_{q}(\mu)
\end{array} .\right.
$$

This approximation of $\mu$ by the probability with finite support $\sum_{q=1}^{Q} \lambda_{q}(\mu) \delta_{x_{q}}$ is such that

$$
d_{W}\left(\mu, \sum_{q=1}^{Q} \mu\left(\Omega_{q}\right) \delta_{x_{q}}\right) \leq \eta, \quad d_{W}\left(\sum_{q=1}^{Q} \mu\left(\Omega_{q}\right) \delta_{x_{q}}, \sum_{q=1}^{Q} \lambda_{q}(\mu) \delta_{x_{q}}\right) \leq \frac{2 Q}{N} .
$$


Now, for each element $\lambda \in \Lambda$, there exists an $\eta$-optimal strategy of P1 in the game $\Gamma_{1}\left(\sum_{q=1}^{Q} \lambda_{q} \delta_{x_{q}}\right)$ denoted $h(\lambda)$, which we can clearly identify to an element of $\Delta(I)^{Q}$ with coordinates $h(\lambda)_{q}$. We are now able to define P1's strategy:

$$
H_{\varepsilon}(L, \mu)=h(\lambda(\mu))_{q} \text { if } L \in \Omega_{q} .
$$

This defines a jointly measurable application and it remains to prove that it guarantees the right quantity. Let us fix $\mu \in \Delta\left(S_{K}\right)$ and a strategy $\tau$ for P2. The triplet $\left(\mu, H_{\varepsilon}(., \mu), \tau\right)$ defines a joint probability $\pi$. The associated payoff is

$$
\begin{aligned}
\mathbb{E}_{\pi} & {[\langle L, A(i, j)\rangle] \geq \mathbb{E}_{\pi}\left[\sum_{q=1}^{Q} \mathbb{I}_{L \in \Omega_{q}}\left\langle x_{q}, A(i, j)\right\rangle\right]-C_{A} \eta } \\
& \left.=\sum_{q=1}^{Q} \mu\right)\left(\Omega_{q}\right)\left\langle x_{q}, \int A(i, j) d\left[h(\lambda(\mu))_{q}(i) \otimes \tau(j)\right]\right\rangle-C_{A} \eta \\
& \geq \sum_{q=1}^{Q} \lambda_{q}(\mu)\left\langle x_{q}, \int A(i, j) d\left[h(\lambda(\mu))_{q}(i) \otimes \tau(j)\right]\right\rangle-\frac{2 Q}{N} C_{A}-C_{A} \eta \\
& \geq \underline{V}_{1}\left(\sum_{q=1}^{Q} \lambda_{q}(\mu) \delta_{x_{q}}\right)-\eta-\frac{2 Q}{N} C_{A}-C_{A} \eta
\end{aligned}
$$

Using that $\underline{V}_{1}$ is $C_{A}$-Lipschitz for the Wasserstein distance $d_{W}$ and the preceding inequalities

$$
\left|\underline{V}_{1}\left(\sum_{q=1}^{Q} \lambda_{q}(\mu) \delta_{x_{q}}\right)-\underline{V}_{1}(\mu)\right| \leq C_{A}\left(\eta+\frac{2 Q}{N}\right),
$$

and finally

$$
\mathbb{E}_{\pi}[\langle L, T(i, j)\rangle] \geq \underline{V}_{1}(\mu)-2 C_{A}\left(\eta+\frac{2 Q}{N}\right)-\eta,
$$

which ends the proof by choosing $\eta$ sufficiently small and $N$ sufficiently large so that $\frac{2 Q}{N} C_{A}$ is bounded by $\eta$.

6.2. Jensen Inequality. The vector space $M^{1}\left(S_{K}\right)$ of finite signed Borel measures $\mu$ on $S_{K}$ is endowed with the weak*-topology. The induced topology on $\Delta\left(S_{K}\right)$ is metrizable by $d_{W}$.

Lemma 6.1. Let $(\Omega, \mathcal{A}, \mathbb{P})$ a probability space, $\mathcal{G} \subset \mathcal{F}$ two sub $\sigma$-algebra of $\mathcal{A}$, and $f$ a concave continuous mapping from $\Delta\left(S_{K}\right)$ to $\mathbb{R}$. Then, for all $S_{K}$-valued random variable $X$ :

- $f(\llbracket X \rrbracket) \geq \mathbb{E}[f(\llbracket X \mid \mathcal{F} \rrbracket)]$

- $f(\llbracket X \mid \mathcal{G} \rrbracket) \geq \mathbb{E}[f(\llbracket X \mid \mathcal{F} \rrbracket) \mid \mathcal{G}]$ almost surely

Proof. Let $\Phi$ denote the distribution of $\llbracket X \mid \mathcal{F} \rrbracket$ in $\Delta\left(\Delta\left(S_{K}\right)\right)$. $f$ being continuous, it is sufficient to prove that $\mu=\llbracket X \rrbracket$ is the barycenter of $\Phi$. But, for all $h \in C\left(S_{K}\right)$, it follows from the properties of the conditional expectation that

$$
\int\langle h, \nu\rangle d \Phi(\nu)=\mathbb{E}[\mathbb{E}[h(X) \mid \mathcal{F}]]=\mathbb{E}[h(X)]=\langle h, \mu\rangle
$$

which proves the first result. The second one follows by the same method. It is sufficient to prove that $\llbracket X \mid \mathcal{G} \rrbracket$ is almost surely the barycenter the $\Delta\left(\Delta\left(S_{K}\right)\right)$-valued $\mathcal{G}$-measurable random variable

$$
\Psi=\llbracket \llbracket X|\mathcal{F} \rrbracket| \mathcal{G} \rrbracket .
$$

Applying the previous argument to a well-chosen countable subset $\mathcal{C}_{0}$ of $C\left(S_{K}\right)$ and by using the definitions of conditional laws and conditional expectations, we have with probability one

$$
\forall h \in \mathcal{C}_{0}, \quad \int\langle h, \nu\rangle d \Psi(\nu)=\mathbb{E}[\mathbb{E}[h(X) \mid \mathcal{F}] \mid \mathcal{G}]=\mathbb{E}[h(X) \mid \mathcal{G}]
$$

Now $\mathcal{C}_{0}$ can be taken as a countable convergence determining subset of $C\left(S_{K}\right)$ so that property (6.1) extends to all $h \in C\left(S_{K}\right)$ and this implies

$$
f(\llbracket X \mid \mathcal{G} \rrbracket) \geq \mathbb{E}[f(\llbracket X \mid \mathcal{F} \rrbracket) \mid \mathcal{G}]
$$

with probability one. 
Disintegration. Let $E, F$ be a compact metric spaces and consider the application

$$
T: \Delta(E \times F) \rightarrow \Delta(\Delta(E))
$$

which associates to the law $\pi$ of a pair $(X, Y)$ of $E \times F$-valued random variables, the law of the conditional law $\llbracket X \mid Y \rrbracket$. Formally $T(\pi)$ is defined as the unique probability such that for every bounded continuous function $f$ on $\Delta(E)$

$$
\int_{\Delta(E)} f(u) d T(\pi)(u)=\mathbb{E}\left[f \left(\llbracket[X \mid Y \rrbracket]=\int_{F} f(\pi(. \mid Y=y)) d \pi_{F}(y)\right.\right.
$$

where $\pi_{F}$ denotes the marginal law of $Y$ and $\pi(. \mid Y=y)$ a version of the conditional law of $X$ given $Y$.

Lemma 6.2. For any convex lower semi-continuous function $f$ on $\Delta(E)$, the application

$$
\pi \rightarrow \int_{\Delta(E)} f(u) d T(\pi)(u)
$$

is convex and lower semi-continuous.

Proof. The main line of this proof is borrowed from the theory of lower semi-continuous relaxation of integral convex functionals in measure spaces (see Buttazzo [10]). At first, up to a translation, we can assume that $f \geq 0$. There exists a countable family $\phi_{n} \in C^{0}(E)$ such that

$$
f(\mu)=\sup _{n \in \mathbb{N}} \widetilde{\phi}_{n}(\mu)=\sup _{n \in \mathbb{N}} \int_{E} \phi_{n}(t) d \mu(t)
$$

Extend $f$ on the whole space of bounded Radon measure over $E, M(E)$, by the value $+\infty$ outside $\Delta(E), f$ being lower semi-continuous and convex is then equal to a supremum of the continuous affine functions. Linear continuous functions are exactly the applications $\mu \rightarrow \int \phi d \mu$ for $\phi \in C^{0}(E)$ by definition of the weak ${ }^{*}$ topology on $M(E)$ and any affine continuous function is equal to a linear mapping on $\Delta(E)$ since the constant can be put in the linear part. We can replace this supremum by a supremum over a countable family using a countable convergence determining subset (see e.g. [2] p106-107). At last, define $\phi_{n, k}$ as the Moreau-Yosida approximation

$$
\forall x \in E, \phi_{n, k}(x)=\inf _{y \in E} \phi_{n}(y)+k d(x, y)
$$

so that by monotone convergence

$$
f(\mu)=\sup _{n \in \mathbb{N}} \sup _{k \in \mathbb{N}} \widetilde{\phi}_{n, k}(\mu) .
$$

Given some $\varepsilon>0$, we can restrict the supremum to functions $\phi_{n, k}$ is such that there exists $\mu \in \Delta(E), \int \phi_{n, k} d \mu \geq$ $f(\mu)-\varepsilon$, the remaining functions being replaced by 0 . Using monotone convergence, it is then sufficient to prove that for any fixed $k$, the function

$$
\pi \rightarrow \int f_{p}(u) d T(\pi)(u)
$$

is convex and lower semi-continuous with

$$
f_{p}(\mu)=\sup _{k \leq p} \sup _{n \in \mathbb{N}} \widetilde{\phi}_{n, k}(\mu)
$$

The functions $\phi_{n, k}$ in the above expression being $p$-Lipschitz, they are uniformly bounded from below by $p \operatorname{diam}(E)-\varepsilon$, and we can assume w.l.o.g. that they are nonnegative. Applying Lemma 3.11 p125 in Buttazzo $[10]$

$$
\begin{aligned}
\int_{F} f_{p}(\pi(. \mid Y=y)) d \pi_{F}(y) & =\sup _{\left(B_{i}\right)_{i=i_{1}, \ldots, i_{p}}} \sum_{i} \int_{B_{i}} \widetilde{\phi}_{i, k_{i}}(\pi(. \mid Y=y)) d \pi_{F}(y) \\
& =\sup _{\left(B_{i}\right)_{i=i_{1}, \ldots, i_{p}}} \sum_{i} \int_{B_{i}} \int_{E} \phi_{i, k_{i}}(t) d \pi(t \mid Y=y) d \pi_{F}(y)
\end{aligned}
$$

where the supremum is over the finite measurable partitions of the space $F$. For $(n, k)$ fixed, consider the application defined on the Borel $\sigma$-field of $F$

$$
B \in \mathcal{B}(F) \rightarrow \mathcal{F}_{n, k}(B)=\int_{B} \int_{E} \phi_{n, k}(t) d \mu(t \mid Y=y) d \pi_{F}(y)=\int_{E \times F} \phi_{n, k}(x) \mathbb{I}_{B}(y) d \pi(x, y)
$$

This is a clearly a bounded positive measure over $F$. $F$ begin compact metric, it is inner regular, i.e.

$$
\mathcal{F}_{n, k}(B)=\left\{\sup \mathcal{F}_{n, k}(K): K \subset B, K \text { compact }\right\}
$$

One can then replace

$$
\sup _{\left(B_{i}\right)_{i=i_{1}, \ldots, i_{p}}} \sum_{i} \mathcal{F}_{i, k_{i}}\left(B_{i}\right)=\sup _{\left(K_{i}\right)_{i=i_{1}, \ldots, i_{p}}} \sum_{i} \mathcal{F}_{i, k_{i}}\left(K_{i}\right)=\sup _{\left(O_{i}\right)_{i=i_{1}, \ldots, i_{p}}} \sum_{i} \mathcal{F}_{i, k_{i}}\left(O_{i}\right)
$$


where the second supremum is over finite families of pairwise disjoint compact subsets and the third over finite families of pairwise disjoint open sets using that the distance between disjoint compact sets is positive. Finally, we just have to remark that for a fixed family of open sets, the mapping

$$
\pi \rightarrow \sum_{i} \mathcal{F}_{i, k_{i}}\left(O_{i}\right)
$$

is linear and lower semicontinuous, which implies that our functional is convex and lower semicontinuous.

Let us end with two Lemmas. The first one is an easy result of convex analysis and the second one will play the role of Proposition 4.4 for the time-dependent extension of our results.

Lemma 6.3. For a convex lower semi-continuous function $\phi$ on $\mathbb{R}^{K}$ with values in $\mathbb{R} \cup\{+\infty\}$, the two following assertions are equivalent

i) The domain of $\phi$ is $S_{K}$ and $\phi$ is Lipschitz on $S_{K}$.

ii) for all $i=1, \ldots, K$, there exists $z_{i} \in \partial \phi\left(e_{i}\right)$ such that $z_{i}+N_{i} \subset \partial \phi\left(e_{i}\right)$ where $N_{i}$ denotes the normal cone of $S_{K}$ at $e_{i}$ :

$$
N_{i}:=\left\{z \in \mathbb{R}^{K} \mid \forall p \in S_{K},\left\langle z, p-e_{i}\right\rangle \leq 0\right\} .
$$

Proof. Assume $i)$. $\phi$ being Lipschitz on $S_{K}, \partial \phi\left(e_{i}\right) \neq \emptyset$. For $z \in \partial \phi\left(e_{i}\right)$, we have

$$
\forall p \in S_{K}, \forall y \in N_{i}, \phi(p)-\phi\left(e_{i}\right) \geq\left\langle z, p-e_{i}\right\rangle \geq\left\langle z+y, p-e_{i}\right\rangle .
$$

It follows that $z+N_{i} \subset \partial \phi\left(e_{i}\right)$ which in turn implies $\left.i i\right)$. Assume now $\left.i i\right)$. At first $S_{K}$ is included in Dom( $\left.\phi\right)$ and $\phi$ is Lipschitz on $S_{K}$ since $\partial \phi\left(e_{i}\right) \neq \emptyset$ for all $i=1, . ., K$. For $x \notin S_{K}$, we have for all $i$ and $y \in N_{i}$,

$$
\phi(x) \geq \phi\left(e_{i}\right)+\left\langle x-e_{i}, z_{i}+y\right\rangle
$$

where $z_{i} \in \partial \phi\left(e_{i}\right)$ is given by $\left.i i\right)$. If there exists $j$ such that $x_{j}<0$, then we can choose $i \neq j$ so that $y=-\lambda e_{j} \in N_{i}$ for all $\lambda>0$, and it follows that $\phi(x)=+\infty$. If $x \geq 0$, then $\alpha=\sum_{j} x_{j}-1 \neq 0$ and for any index $i$ and any $\lambda>0, \lambda \alpha\left(\sum_{j} e_{j}\right) \in N_{i}$ and we conclude that $\phi(x)=+\infty$.

The following proof is a direct adaptation of the proof of Proposition 4.1 in [11].

Lemma 6.4. Define

$$
V(\mu):=\sup _{\llbracket\left(X_{t}\right)_{t \in[0,1] \rrbracket \in \mathcal{M}(\preceq \mu)}} \mathbb{E}\left[\int_{0}^{1} \bar{u}\left(s, X_{s}\right) d s\right] .
$$

Then $V(\mu)=\inf _{\phi \in E} W(\phi)+\int \phi d \mu$ where $E$ denotes the set of convex and $C_{A}$-Lipschitz functions on $S_{K}$.

Proof. Given $\phi \in E$, define for $(t, p) \in[0,1] \times S_{K}$

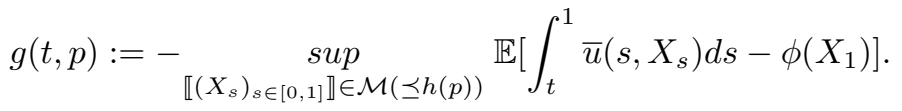

Then $g$ is a viscosity solution in the dual sense of the Hamilton-Jacobi equation (see [12])

$$
\frac{\partial g}{\partial t}(t, p)-\bar{u}(t, p)=0
$$

with terminal condition $g(1, p)=\phi(p)$. Note at first that $g(t,$.$) is convex in p$ (using that $h$ is linear and $\preceq$ is convex) and that the terminal condition is met since $\phi$ is convex. Let $f$ be a $C^{1}$ function on $[0,1]$ such that $f-g(., p) \geq 0$ with equality in $t$ for some $p \in S_{K}$. Then, for all $h>0$, we can replace any martingale $X$ in $\mathcal{M}(h(p))$ by the martingale equal to $p$ for $s<t+h$ and to $X_{s}$ for $s \geq t+h$. It follows that

$$
f(t)=g(t, p) \leq-\int_{t}^{t+h} \bar{u}(s, p) d s+g(t+h, p) \leq-\int_{t}^{t+h} \bar{u}(s, p) d s+f(t+h) .
$$

We obtain $f^{\prime}(t)-\bar{u}(t, p) \geq 0$ which proves that $g$ is a dual subsolution of (6.2).

Let $\psi$ denote the convex conjugate of $g$, then

$$
\psi(t, z):=\sup _{p \in S_{K}}\langle p, z\rangle-g(t, p)=\sup _{\llbracket\left(X_{t}\right)_{t \in[0,1]} \rrbracket \in \mathcal{M}} \mathbb{E}\left[\left\langle z, X_{t}\right\rangle+\int_{t}^{1} \bar{u}\left(s, X_{s}\right) d s-\phi\left(X_{1}\right)\right],
$$

where $\mathcal{M}:=\cup_{\mu \in \Delta\left(S_{K}\right)} \mathcal{M}(\preceq \mu)$. It is easily seen that $\psi$ is globally continuous, convex and 1-Lipschitz with respect to $z$, and that $\psi(1, z)=\phi^{*}(z)$. Now, let $X$ be optimal for $\psi(t, z)$, i.e. such that

$$
\psi(t, z)=\mathbb{E}\left[\left\langle z, X_{t}\right\rangle+\int_{t}^{1} \bar{u}\left(s, X_{s}\right) d s-\phi\left(X_{1}\right)\right] .
$$

Note that the conditional law of $\left(X_{t \vee s}\right)_{s \in[0,1]}$ given $X_{t}$ is in $\mathcal{M}$ with probability 1 so that

$$
\psi(t, z)=\mathbb{E}\left[\left\langle z, X_{t}\right\rangle+\int_{t}^{1} \bar{u}\left(t, X_{t}\right) d t-\phi\left(X_{1}\right) \mid X_{t}\right] .
$$


Moreover, for all $y \in \mathbb{R}^{K}$, the following inequalities hold almost surely

$$
\psi(t, z+y) \geq \mathbb{E}\left[\left\langle z+y, X_{t}\right\rangle+\int_{t}^{1} \bar{u}\left(t, X_{t}\right) d t-\phi\left(X_{1}\right) \mid X_{t}\right]=\left\langle y, X_{t}\right\rangle+\psi(t, z),
$$

which implies that $X_{t} \in \partial \psi(t, z)$ where $\partial \psi(t, z)$ denotes the subdifferential of $\psi(t,$.$) .$

Let $f$ be a $C^{1}$ function on $[0,1] \times \mathbb{R}^{K}$ such that $f-\psi \geq 0$ with equality in $(t, z)$. It follows that $\psi(t,$.$) is$ differentiable in $z$. Let $x:=\nabla \psi(t, z)=\nabla f(t, z)$ and let $X$ be optimal in $\psi(t, z)$, then using the above result, $X_{t}=x$ almost surely, and

$$
f(t, z)=\psi(t, z) \leq \mathbb{E}\left[\int_{t}^{t+h} \bar{u}\left(s, X_{s}\right) d s\right]+\psi(t+h, z) \leq \mathbb{E}\left[\int_{t}^{t+h} \bar{u}\left(s, X_{s}\right) d s\right]+f(t+h, z) .
$$

Using that the trajectories of $X$ are càdlàg, we obtain

$$
\lim _{h \rightarrow 0+} \frac{1}{h} \mathbb{E}\left[\int_{t}^{t+h} \bar{u}\left(s, X_{s}\right) d s\right]=\bar{u}(t, x) .
$$

This implies that $\frac{\partial f}{\partial t}(t, z)+\bar{u}(t, x) \geq 0$, which proves that $\psi$ is a subsolution of (5.2), and implies that $g$ is a dual supersolution of $(6.2)$.

Let $\chi$ be the unique viscosity solution of (5.2). At first, using that $\psi$ is a subsolution of (5.2), we have by standard comparison Theorems (see e.g. [16]) that $\chi \geq \psi$. Note then that for all $t, x \rightarrow \chi(t, x)$ is convex as a limit of convex functions using Lemma 4.2 and Proposition 4.3. Let $\omega(t,$.$) denote the convex conjugate of$ $\chi(t,$.$) . Using the preceding Lemma, we will prove that \operatorname{Dom}(\omega(t,))=.S_{K}$ and $\omega(t,$.$) is Lipschitz on S_{K}$. To see this, note that from Proposition 4.3, $\chi(t, x)$ depends only on the restriction of $\phi^{*}$ to the ball $B\left(x, C_{A}\right)$ and that $\phi$ fulfils the property $i$ ) of Lemma 6.3 by assumption. Let $z_{i} \in \partial \phi\left(e_{i}\right)$ given by property $\left.i i\right)$ of Lemma 6.3 and choose $y_{i}$ such that $y_{i}+N_{i}+B\left(0, C_{A}\right) \subset \partial \phi\left(e_{i}\right)$, which always exists using $\left.i i\right)$ and since $N_{i}$ is a convex cone with non-empty interior. Using Fenchel's Lemma, $e_{i} \in \partial \phi^{*}(z)$ for all $z \in \partial \phi\left(e_{i}\right)$, so that $\phi^{*}(z)=c_{i}+\left\langle e_{i}, z\right\rangle$ for some constant $c_{i}$. The (classical) solution of equation 5.2 with terminal condition $c_{i}+\left\langle e_{i}, z\right\rangle$ is given by $\psi_{i}(t, z)=c_{i}+\int_{t}^{1} \bar{u}\left(s, e_{i}\right) d s+\left\langle e_{i}, z\right\rangle$. It follows that $\chi=\psi_{i}$ on $[0,1] \times\left(y_{i}+N_{i}\right)$. This implies that for all $t$, $e_{i} \in \partial \chi(t, z)$ for $z \in y_{i}+N_{i}$, and thus that $y_{i}+N_{i} \subset \partial \omega\left(t, e_{i}\right)$ which proves that $\operatorname{Dom}(\omega(t,))=.S_{K}$ and $\omega(t,$. is Lipschitz on $S_{K}$.

Finally, $\chi$ being subsolution of (5.2), this implies that $\omega$ is a dual supersolution of (6.2). We conclude that $\omega \geq g$ so that $\chi \leq \psi$ using the comparison Theorem given in [12]. Together with the preceding inequality, we obtain $\psi=\chi$ and thus $\omega=g$. As a consequence, for all $\phi \in E$,

$$
W(\phi)=\sup _{p \in \Delta(K)} g(0, p)=\sup _{\mu \in \Delta\left(S_{K}\right)} V(\mu)-\int \phi d \mu .
$$

As in Lemma 4.6, for any $\phi \in C\left(S_{K}\right)$, one can define $\tilde{\phi} \in E$ such that $\tilde{\phi} \leq \phi$ and $W(\phi)=W(\tilde{\phi})$. $V$ being concave and weakly upper semi-continuous, it is equal to its bi-conjugate and we obtain

$$
V(\mu)=\inf _{\phi \in C\left(S_{K}\right)} W(\phi)+\int \phi d \mu=\inf _{\phi \in E} W(\phi)+\int \phi d \mu
$$

which concludes the proof.

Acknowledgement 1. The author gratefully acknowledges the support of the Agence Nationale de la Recherche, under grant ANR JEUDY, ANR-10-BLAN 0112.

\section{REFERENCES}

[1] Barron E.N. Alvarez O. and Ishii H. Hopf-Lax formulas for semicontinuous data. Indiana Univ. Math. J., 48:993-1036, 1999.

[2] L. Ambrosio, N. Gigli, and G. Savaré. Gradient flows in metric spaces and in the space of probability measures. Birkhauser, 2008.

[3] R.J. Aumann. Mixed and Behavior Strategies in Infinite Extensive Games. Advances in game theory, 1964.

[4] R.J. Aumann, M. Maschler, and R.E. Stearns. Repeated games with incomplete information. The MIT press, 1995.

[5] M. Bardi and L.C. Evans. On Hopf's formulas for solutions of Hamilton-Jacobi equations. Nonlinear analysis, 8(11):1373-1381, 1984.

[6] D.P. Bertsekas and S.E. Shreve. Stochastic optimal control: The discrete time case. Academic Press, 1978.

[7] D. Blackwell. Equivalent comparisons of experiments. The Annals of Mathematical Statistics, 24(2):265-272, 1953.

[8] D. Blackwell. An analog of the minimax theorem for vector payoffs. Pacific Journal of Mathematics, 6(1):1-8, 1956.

[9] D. Blackwell and L.E. Dubins. An extension of Skorohod's almost sure representation theorem. Proceedings of the American Mathematical Society, 89(4):691-692, 1983.

[10] G. Buttazzo, M. Giaquinta, and S. Hildebrandt. One-dimensional variational problems: an introduction. Oxford University Press, USA, 1998.

[11] P. Cardaliaguet and C. Rainer. On a continuous-time game with incomplete information. Math. Oper. Res., 34(4):769-794, 2009 .

[12] P. Cardaliaguet and C. Rainer. Stochastic differential games with asymmetric information. Applied Mathematics and Optimization, 59:1-36, 2009. 
[13] P. Cardaliaguet and C. Rainer. Games with incomplete information in continuous time and for continuous types. Dynamic Games and Applications, 2:206-227, 2012.

[14] Laraki R. Cardaliaguet P. and Sorin S. A continuous time approach for the asymptotic value in two-person zero-sum repeated games. SIAM J. Control. Optim., to appear.

[15] G. Choquet. Lectures on analysis I-III. New York: W.A. Benjamin, 1969.

[16] Lions P.-L. Crandall M.G., Ishii H. User's guide to viscosity solutions of second order Partial Differential Equations. Bull. Amer. Soc., 27:1-67, 1992.

[17] B. De Meyer. Repeated games and partial differential equations. Math. Oper. Res., 21(1):209-236, 1996.

[18] B. De Meyer. Repeated games, duality and the central limit theorem. Math. Oper. Res., 21(1):237-251, 1996.

[19] B. De Meyer. Price dynamics on a stock market with asymmetric information. Games Econom. Behav., 69(1):42-71, 2010.

[20] B. De Meyer, E. Lehrer, and D. Rosenberg. Evaluating Information in Zero-Sum Games with Incomplete Information on Both Sides. Math. Oper. Res., 35(4):851, 2010.

[21] B. De Meyer and D. Rosenberg. "Cav u" and the Dual Game. Math. Oper. Res., 24(3):619-626, 1999.

[22] K. Fan. Minimax theorems. Proceedings of the National Academy of Sciences of the United States of America, 39(1):42, 1953.

[23] F. Gensbittel. Covariance control problems over martingale arising from game theory. preprint, 2011.

[24] E. Kohlberg. Optimal strategies in repeated games with incomplete information. Internat. J. Game Theory, 4(1):7-24, 1975.

[25] R. Laraki. Repeated games with lack of information on one side: the dual differential approach. Math. Oper. Res., 27(2):419440, 2002.

[26] J.-F. Mertens, S. Sorin, and S. Zamir. Repeated games. CORE Discussion Papers 9420, 9421 and 9422, Universite Catholique De Louvain, Belgium, 1994.

[27] P.A. Meyer and W.A. Zheng. Tightness criteria for laws of semimartingales. Annales de l'Institut Henri Poincare (B) Probability and Statistics, 20(4):353-372, 1984

[28] J. Neveu. Bases mathématiques du calcul des probabilités. Masson, Paris, 1970.

[29] S. Sorin. A first course on zero-sum repeated games. Springer, 2002.

[30] P.E. Souganidis. Approximation schemes for viscosity solutions of Hamilton-Jacobi equations. Journal of differential equations, $59(1): 1-43,1985$.

Université Toulouse 1 Capitole, Manufacture des Tabacs, Office MF213, 21, Allée de Brienne 31015 Toulouse Cedex 6.

E-mail address: fabien.gensbittel@ut-capitole.fr 